\title{
Data compression with low distortion and finite blocklength
}

\author{
Victoria Kostina
}

\begin{abstract}
This paper considers lossy source coding of $n$-dimensional continuous memoryless sources with low distortion and shows a simple, explicit approximation to the minimum source coding rate. More precisely, a nonasymptotic version of Shannon's lower bound is presented. Lattice quantizers are shown to approach that lower bound, provided that the source density is smooth enough and the distortion is low, which implies that fine multidimensional lattice coverings are nearly optimal in the rate-distortion sense even at finite $n$. The achievability proof technique avoids both the usual random coding argument and the simplifying assumption of the presence of a dither signal.

The paper also presents a necessary and sufficient condition for Shannon's lower bound to be attained exactly. Although most continuous sources violate that condition, all finite alphabet sources satisfy it at low distortion levels. The rate-dispersion function is then given simply by the varentropy of the source.
\end{abstract}

\section{Index Terms}

Lossy source coding, lattice coding, rate-distortion function, Shannon's lower bound, low distortion, high resolution, finite blocklength regime, dispersion.

\section{INTRODUCTION}

We showed in [2] that for the compression of a memoryless source with single-letter distribution $P_{\mathrm{X}}$ under a single-letter distortion measure $\mathrm{d}(\cdot, \cdot)$, the minimum achievable source coding rate $R(n, d, \epsilon)$ comparable with blocklength $n$ and the probability $\epsilon$ of exceeding distortion $d$ given by

$$
R(n, d, \epsilon)=R(d)+\sqrt{\frac{\mathcal{V}(d)}{n}} Q^{-1}(\epsilon)+O\left(\frac{\log n}{n}\right),
$$

V. Kostina is with California Institute of Technology (e-mail: vkostina@caltech.edu). A part of this paper was presented at the 2015 Allerton conference [1]. 
where $Q$ is the complementary Gaussian cdf, $R(d)$ is the rate-distortion function of the source:

$$
R(d)=\mathbb{R}_{\mathrm{X}}(d) \triangleq \inf _{\substack{P_{\mathrm{Y} \mid \mathrm{X}}: \\ \mathbb{E}[\mathrm{d}(\mathrm{X}, \mathrm{Y})] \leq d}} I(\mathrm{X} ; \mathrm{Y}),
$$

and $\mathcal{V}(d)$ is a parameter we termed the rate-dispersion function. That parameter quantifies the overhead over the rate-distortion function incurred by the finite blocklength constraint. Dropping the remainder term in (1), we obtain a simple approximation to the minimum achievable coding rate. That approximation provides good accuracy even at short blocklengths, as evidenced by the numerical results in [2].

In this contribution, we derive a simplification of (1) in the regime of low $d$. The interest in pursuing such a simplification stems from the fact that closed-form formulas for $R(d)$ and $\mathcal{V}(d)$ are rarely available. Indeed, the rate-distortion and the rate-dispersion function are given by the mean and the variance of $\jmath_{\mathrm{X}}(\mathrm{X}, d)$, d-tilted information, the random variable which is defined as

$$
\jmath \mathbf{x}(\mathrm{x}, d) \triangleq \log \frac{1}{\mathbb{E}\left[\exp \left\{\lambda^{\star} d-\lambda^{\star} \mathrm{d}\left(\mathrm{x}, \mathrm{Y}^{\star}\right)\right\}\right]},
$$

where $\lambda^{\star}=-\mathbb{R}_{X}^{\prime}(d)$, and the expectation is with respect to the unconditional distribution of $Y^{\star}$, the random variable that attains the rate-distortion function, i.e. $\mathbb{R}_{\mathbf{X}}(d)=I\left(\mathrm{X} ; \mathrm{Y}^{\star}\right)$. Thus, both the rate-dispersion and the rate-distortion function are described in terms of the solution to the convex optimization problem in (2). Although the convexity of the problem in (2) often allows for an efficient numerical computation of its optimum [3], closed-form expressions are available only in special cases. In those cases, the distortion measure is carefully tailored to match the source.

By a fortuitous coincidence, not only is the high resolution regime prevalent in practice of data compression, but it also allows for a vast simplification both in the analysis of the fundamental tradeoffs in data compression and in coding strategies to attain those tradeoffs.

This paper shows that under regularity conditions, the d-tilted information in a random variable $X \in \mathbb{R}^{n}$ satisfies

$$
\jmath_{X}(X, d) \approx \log \frac{1}{f_{X}(X)}-\phi(d) \quad \text { w.h.p. }
$$

as long as $d$ is small enough, where $f_{X}$ is the source density, and $\phi(d)$ is a term that depends only on the distortion measure and distortion threshold $d$. For example, for mean-square error (MSE) distortion,

$$
\phi(d)=n \log \sqrt{2 \pi e d} .
$$

For the compression of finite alphabet sources under permutation distortion measures, an even stronger claim holds, namely,

$$
\jmath_{X}(X, d)=\log \frac{1}{P_{X}(X)}-\phi(d) \quad \text { a.s. }
$$


as long as $d \leq d_{0}$, where $d_{0}>0$ is a function of $P_{X}$ only.

The value of $\jmath_{X}(x, d)$ can be loosely interpreted as the amount of information that needs to be stored about $x$ in order to restore it with distortion $d$ [2]. The explicit nature of (4) illuminates the tension between $f_{X}(X)$ and the target distortion: the likelier $X$ is, the fewer bits are required to store it; the lower tolerable $d$ is, the more bits are required in order to represent the source with that distortion. We stress that this intuitively pleasing insight is not afforded by the general formula (3).

To gain further understanding of the form of (4), recall that Shannon's lower bound [4] states that the rate-distortion function is bounded below by the difference between the differential entropy of the source, and a term that depends only on the distortion measure and distortion threshold $d$. For example, for mean-square error distortion, Shannon's lower bound is given by

$$
\mathbb{R}_{X}(d) \geq \underline{\mathbb{R}}_{X}(d) \triangleq h(X)-\phi(d)
$$

where $h(X)$ is the differential entropy of the source. Due to its simplicity and because it becomes increasingly tight in the limit of low distortion [5], [6], $\mathbb{R}_{X}(d)$ is often used as a convenient proxy for $\mathbb{R}_{X}(d)$. The statement in (4) can be viewed as a nonasymptotic refinement of those results. More precisely, this paper shows a nonasymptotic version of Shannon's converse bound and demonstrates that it can be approached by a lattice quantizer followed by a lossless coder. A careful analysis of those bounds reveals that for a class of difference distortion measures and memoryless sources with sufficiently smooth densities, as $d \rightarrow 0$ and $n \rightarrow \infty$, the nonasymptotically achievable source coding rate admits the following expansion:

$$
R(n, d, \epsilon)=\underline{R}(d)+\sqrt{\frac{\underline{\mathcal{V}}}{n}} Q^{-1}(\epsilon)+O(\sqrt{d})+O\left(\frac{\log n}{n}\right),
$$

where

$$
\underline{\mathcal{V}} \triangleq \operatorname{Var}\left[\log f_{\mathbf{X}}(\mathrm{X})\right]
$$

is the variance of the right side of (4).

Since (8) is attained by lattice quantization, lattice quantizers are nearly optimal at high resolution even at finite blocklength. The implication for engineering practice is that, in the search for good codes, it is unnecessary to consider more complex structures than lattices if the goal is high resolution digital representation of the original analog signal. Due to the regularity of the code vector locations, lattice quantizers offer a great reduction in the complexity of encoding algorithms (e.g. [7], [8]). Therefore, both their performance and their regular algebraic structure make lattices a particularly appealing choice for efficient analog-to-digital conversion. 
This paper also studies the asymptotic rate-distortion performance of lattice quantization of continuous ergodic sources. In particular, we prove that lattice quantization attains Shannon's lower bound in the limit, even if the source is nonstationary. For stationary ergodic sources satisfying a regularity assumption, we show that Shannon's lower bound is still attained at speed $O\left(\frac{1}{\sqrt{n}}\right)$ with increasing blocklength.

Notable prior contributions to the understanding of lattice quantizers in large dimension include the works by Rogers [9], Gersho [10], Zamir and Feder [11] and Linder and Zeger [12]. Rogers [9] showed the existence of efficient lattice coverings of space. Using a heuristic approach, Gersho [10] studied tessellating vector quantizers, i.e. quantizers whose regions are congruent with some tessellating convex polytope $P .{ }^{1}$ Although every lattice quantizer is a tessellating quantizer, the converse is not true. Gersho [10] showed heuristically that in the limit of low distortion, tessellating vector quantizers approach $n$-dimensional Shannon's lower bound. Relying on a conjecture by Gersho, Linder and Zeger [12] streamlined the proof of Gersho's result and reported that the minimum entropy among all $n$ dimensional tessellating vector quantizers approaches the $n$-letter Shannon's lower bound in the limit of low $d$, provided that the Gersho conjecture is true. Zamir and Feder [11] considered the setting in which a signal called a dither is added to an input signal prior to quantization, namely, dithered quantization, and showed an upper bound on the achievable conditional (on the dither) output entropy of dithered lattice quantizers. Their result relied on a rather restrictive assumption on the source density violated even by the Gaussian distribution. That assumption was later relaxed by Linder and Zeger [12]. While the assumption of the availability of the dither signal both at the encoder and the decoder greatly simplifies the analysis and also improves the performance somewhat by smoothening the underlying densities, it can also substantially and unnecessarily complicate the engineering implementation. This paper does not consider dithered quantization.

Historically, theoretical performance analysis of lossy compressors proceeded in two disparate directions: bounds derived from Shannon theory [13], and bounds derived from high resolution approximations [14], [15]. The former provides asymptotic results as the sources are coded using longer and longer blocks. The latter assumes fixed input block size but estimates the performance as the encoding rate becomes arbitrarily large. This paper fuses the two approaches to study the performance of block compressors with high resolution from the Shannon theory viewpoint.

So as not to clutter notation, in those statements in which the Cartesian structure of the space is

\footnotetext{
${ }^{1}$ A polytope $P$ is called tessellating if there exists a partition of $\mathbb{R}^{n}$ consisting of translated and rotated copies of $P$.
} 
unimportant, we will denote random vectors simply by $X, Y$, etc., omitting dimensionality parameter $n$. Wherever necessary, we will make the dimensionality explicit, writing $X^{n}, Y^{n}$ in lieu of $X, Y$. For a stationary memoryless process $X_{1}, X_{2}, \ldots$, we denote by $\mathrm{X}$ the random variable that is distributed the same as $X_{i}, i=1,2, \ldots$. All logarithms are arbitrary common base.

The rest of the paper is organized as follows. Section II discusses Shannon's lower bound, presents the necessary and sufficient condition for it to be attained with equality and proves (6). Section III presents a bound on the output entropy of lattice quantizers. Focusing on the MSE distortion, Section IV introduces the lattice rate-distortion function, while Section V presents the nonasymptotic analysis of non-dithered lattice quantization. Generalization to non-MSE distortion measures is discussed in Section VI.

\section{SHANNON'S LOWER BOUND}

The (informational) rate-distortion function is defined for random variable $X \in \mathcal{X}$ and distortion measure $\mathrm{d}: \mathcal{X} \times \mathcal{X} \mapsto \mathbb{R}_{+}$as the solution to the convex optimization problem:

$$
\mathbb{R}_{X}(d) \triangleq \inf _{\substack{P_{Y \mid X}: \mathcal{X} \mapsto \mathcal{X} \\ \mathbb{E}[\mathrm{d}(X, Y)] \leq d}} I(X ; Y) .
$$

It admits the following parametric representation.

Theorem 1 (Parametric representation of $\left.\mathbb{R}_{X}(d)[16]\right)$. Suppose that the following basic assumptions are satisfied.

(a) $\mathbb{R}_{X}(d)$ is finite for some $d$, i.e. $d_{\min }<\infty$, where

$$
d_{\min } \triangleq \inf \left\{d: \mathbb{R}_{X}(d)<\infty\right\}
$$

(b) The distortion measure is such that there exists a finite set $E \subset \mathcal{X}$ such that

$$
\mathbb{E}\left[\min _{y \in E} \mathrm{~d}(X, y)\right]<\infty
$$

For each $d>d_{\min }$, it holds that

$$
\mathbb{R}_{X}(d)=\max _{g(x), \lambda}\{-\mathbb{E}[\log g(X)]-\lambda d\}
$$

where the maximization is over $g(x) \geq 0$ and $\lambda \geq 0$ satisfying the constraint

$$
\mathbb{E}\left[\frac{\exp (-\lambda \mathrm{d}(X, y))}{g(X)}\right] \leq 1 \forall y \in \mathcal{X}
$$

Remark 1. The maximization over $g(x) \geq 0$ in (13) can be restricted to only $0 \leq g(x) \leq 1$ [16]. 
Remark 2. The d-tilted information (defined in (3), [2]) can be defined as

$$
\jmath_{X}(X, d)=-\log g(X)-\lambda d,
$$

where $(g(\cdot), \lambda)$ attain the maximum in (13). So,

$$
\mathbb{R}_{X}(d)=\mathbb{E}\left[\jmath_{X}(X, d)\right] .
$$

We stress that (13) does not follow simply from writing out the convex dual problem to (10); its proof requires representing (10) as a double minimization and carefully studying its properties. For finite alphabet sources, a parametric representation of $\mathbb{R}_{X}(d)$ is contained in Shannon's paper [4]; Berger's book [17] contains parametric representations of $\mathbb{R}_{X}(d)$ both for discrete and continuous sources. However, it was Csiszár [16] who gave rigorous proofs of (13) in the following much more general setting: $X$ belongs to a general abstract probability space, and the existence of the conditional distribution $P_{Y^{\star} \mid X}$ attaining $\mathbb{R}_{X}(d)$ is not required.

While the original derivation of Shannon's lower bound follows a different approach [13], in fact it can be obtained via (13). ${ }^{2}$ Indeed, each choice of $\lambda \geq 0$ and $g$ satisfying (14) gives rise to a lower bound to $\mathbb{R}_{X}(d)$. Shannon's lower bound corresponds to a particular choice of $(\lambda, g)$.

Shannon's lower bound applies to distortion measures of form,

$$
\mathrm{d}(x, y)=\mathrm{d}(x-y)
$$

and random variables $X$ that belong to one of the following classes:

A. $X$ is supported on a discrete group $\mathcal{X}$.

B. $X \in \mathbb{R}^{n}$ has a density.

For the discrete case, the difference in (17) is understood as the corresponding group operation: $x-y=x \oplus(-y)$. Group structure is needed here solely to ensure that for all $x \in \mathcal{X}$ and all $y \in \mathcal{X}$, $x-y \in \mathcal{X}$.

Table I summarizes the declarations necessary to compute Shannon's lower bound for $X$.

It is easy to verify that the choice of $\lambda$ and $g$ in Table I satisfies (14) (with equality). Shannon's lower bound can thus be stated as follows.

Theorem 2 (Shannon's lower bound [4]). Assume that the distortion measure satisfies (17), and that $X$ belongs to either class in Table I. For all $d \geq d_{\min }$,

$$
\mathbb{R}_{X}(d) \geq \underline{\mathbb{R}}_{X}(d),
$$

${ }^{2}$ Berger [17], in particular, presents a derivation through the parametric representation. 


\begin{tabular}{c|c}
$\mathcal{X}$ is discrete group, $X \in \mathcal{X}$ & $X \in \mathbb{R}^{n}$ is continuous \\
\hline$P_{Z_{\lambda}}(z) \triangleq \frac{\exp (-\lambda \mathrm{d}(z))}{\sum_{z \in \mathcal{X}} \exp (-\lambda \mathrm{d}(z))}$ & $f_{Z_{\lambda}}(z) \triangleq \frac{\exp (-\lambda \mathrm{d}(z))}{\int_{\mathbb{R}^{n}} \exp (-\lambda \mathrm{d}(z)) d z}$ \\
\hline$\phi(d) \triangleq h\left(Z_{\lambda}\right)$ & $\phi(d) \triangleq H\left(Z_{\lambda}\right)$ \\
$=\log \int_{\mathbb{R}^{n}} \exp (\lambda d-\lambda \mathrm{d}(z)) d z$ & $=\log \sum_{z \in \mathcal{X}} \exp (\lambda d-\lambda \mathrm{d}(z))$ \\
\hline$g(x)=P_{X}(x) \exp (\phi(d)-\lambda d)$ & $g(x)=f_{X}(x) \exp (\phi(d)-\lambda d)$ \\
\hline$\underline{\jmath}{ }_{X}(x, d) \triangleq \log \frac{1}{P_{X}(x)}-\phi(d)$ & $\underline{\jmath} X(x, d) \triangleq \log \frac{1}{f_{X}(x)}-\phi(d)$ \\
\hline \multicolumn{2}{c}{$\lambda>0:$ solution to equation $\mathbb{E}\left[\mathrm{d}\left(Z_{\lambda}\right)\right]=d$} \\
\hline
\end{tabular}

TABLE I: Computing Shannon's lower bound.

where

$$
\underline{\mathbb{R}}_{X}(d) \triangleq \mathbb{E}\left[\underline{\jmath}_{X}(X, d)\right]
$$

and $\underline{\jmath}_{X}(x, d)$ is defined in Table I.

The representation in (19) parallels the representation of rate-distortion function through the expectation of d-tilted information in (16).

Example. Let $X^{n}$ be a continuous real-valued random vector. Let $\mathrm{d}$ be the mean-square error distortion:

$$
\mathrm{d}\left(x^{n}, y^{n}\right)=\frac{1}{n} \sum_{i=1}^{n}\left(x_{i}-y_{i}\right)^{2} .
$$

A straightforward calculation reveals that,

$$
\begin{aligned}
\lambda & =\frac{n}{2 d} \log e \\
\phi(d) & =\frac{n}{2} \log d+\frac{n}{2} \log (2 \pi e),
\end{aligned}
$$

so

$$
\underline{\mathbb{R}}_{X^{n}}(d)=h\left(X^{n}\right)+\frac{n}{2} \log \frac{1}{d}-\frac{n}{2} \log (2 \pi e) .
$$

Theorem 2 tacitly assumes that there exists solution $\lambda>0$ to

$$
\mathbb{E}\left[Z_{\lambda}\right]=d
$$


as Table I suggests. A crucial question is under which conditions this solution exists. Toward this end, we show that if

$$
\mathrm{d}(0)=0, \quad \mathrm{~d}(z)>0, z \neq 0
$$

and $|\mathcal{X}|=m$, then (24) has a solution for all $d \in\left(0, \mathbb{E}\left[\mathrm{d}\left(Z_{0}\right)\right]\right]$. Indeed, observe using (25) that

$$
\begin{aligned}
\mathbb{E}\left[\mathrm{d}\left(Z_{0}\right)\right] & =\frac{1}{m} \sum_{z \in \mathcal{X}} \mathrm{d}(z) \\
\lim _{\lambda \rightarrow+\infty} \mathbb{E}\left[\mathrm{d}\left(Z_{\lambda}\right)\right] & =0 .
\end{aligned}
$$

Since $\mathbb{E}\left[\mathrm{d}\left(Z_{\lambda}\right)\right]$ is continuous as a function of $\lambda$ on $[0,+\infty)$, it follows that (24) has a solution for all $d \in\left(0, \mathbb{E}\left[\mathrm{d}\left(Z_{0}\right)\right]\right]$.

For continuous $X$, Linkov [5, Lemma 1] showed that (24) has a unique solution for all sufficiently small $d$, as long as $\mathrm{d}(\cdot)$ satisfies the following mild regularity conditions:

(28) $\mathrm{d}(r)=0$ only at $r=0$, and $\mathrm{d}(r)$ is nondecreasing.

(29) Exists such $\nu>0$ that $\lim _{r \rightarrow 0} r^{-\nu} \mathrm{d}(r)<\infty$.

(30) $\int_{\mathbb{R}_{+}} \mathrm{d}^{2}(r) \exp (-\mathrm{d}(r)) d r<\infty$.

The following result pins down the necessary and sufficient condition for equality in (18) to hold.

Theorem 3. The rate-distortion function is equal to Shannon's lower bound,

$$
\mathbb{R}_{X}(d)=\underline{\mathbb{R}}_{X}(d),
$$

if an only if there exists a random variable $Y$, independent of $Z_{\lambda}$, such that

$$
X=Y+Z_{\lambda}
$$

Moreover, (31) holds if and only if

$$
\jmath_{X}(X, d)=\underline{\jmath}_{X}(X, d), \quad \text { a.s. }
$$

Proof: We prove the theorem for the discrete case. The continuous case is analogous. To show the sufficiency of (32) for (31) to hold, notice that for $Y$ in (32) we have,

$$
\begin{aligned}
\mathbb{R}_{X}(d) & \leq I(X ; Y) \\
& =H(X)-H\left(Z_{\lambda}\right) \\
& =\underline{\mathbb{R}}_{X}(d) .
\end{aligned}
$$


To show the necessity of (32) for (31) to hold, first note that

$$
\begin{aligned}
\max _{P_{Z}: \mathbb{E}[\mathrm{d}(Z)] \leq d} H(Z) & =H\left(Z_{\lambda}\right) \\
& =\phi(d),
\end{aligned}
$$

as can be easily verified by solving the convex optimization problem on the left side of (37).

Second, we show that $\phi(d)$ is strictly concave in $d$. Let $Z_{\lambda_{1}}$ and $Z_{\lambda_{2}}$ attain the maximum in (37) for $d_{1}$ and $d_{2}$, respectively. For $0<\alpha<1$, define the distribution

$$
P_{\bar{Z}} \triangleq \alpha P_{Z_{1}}+(1-\alpha) P_{Z_{2}}
$$

By strict concavity of entropy,

$$
H(\bar{Z})<\alpha H\left(Z_{1}\right)+(1-\alpha) H\left(Z_{2}\right)
$$

Therefore,

$$
\begin{aligned}
\phi(d) & \geq H(\bar{Z}) \\
& >\alpha \phi\left(d_{1}\right)+(1-\alpha) \phi\left(d_{2}\right) .
\end{aligned}
$$

Together, (42) and (37) imply that $\phi(d)$ is strictly increasing in $d$.

Last, observe that by assumption (31),

$$
\begin{aligned}
\mathbb{R}_{X}(d) & =H(X)-\max _{\substack{P_{Y \mid X}: \\
\mathbb{E}[\mathrm{d}(X-Y)] \leq d}} H(X \mid Y) \\
& =H(X)-\phi(d)
\end{aligned}
$$

On the other hand,

$$
\begin{aligned}
\max _{\substack{P_{Y \mid X}: \\
\mathbb{E}[\mathrm{d}(X-Y)] \leq d}} H(X \mid Y) & \leq \max _{\substack{P_{Y}, \delta: \mathcal{X} \mapsto \mathbb{R}^{+} \\
\mathbb{E}[\delta(Y)] \leq d}} \sum_{i \in \mathcal{X}} P_{Y}(i) \max _{\substack{P_{X \mid Y=i}: \\
\mathbb{E}[\mathrm{d}(X-Y) \mid Y=i] \leq \delta(i)}} H(X \mid Y=i) \\
& =\max _{\substack{\left.\delta: \mathcal{X} \mapsto \mathbb{R}^{+}: \\
P_{Y}, \delta[\delta)\right] \leq d}} \mathbb{E}[\phi(\delta(Y))] \\
& \leq \phi(d),
\end{aligned}
$$

where (46) is due to (37), and (47) holds because $\phi(\cdot)$ is concave and increasing. Since $\phi(\cdot)$ is strictly concave, equality in (47) is attained if and only if $\delta(Y) \equiv d$. For this choice of $\delta(Y)$,

$$
P_{X \mid Y=i}=P_{Z_{\lambda}}, i \in \mathcal{X}
$$


equality in (47) holds regardless of the choice of $P_{Y}$ in the right side of (45). If there exist such $P_{Y}$ that (32) holds, then, and only then, the inequality in (45) is satisfied with equality. Therefore, (32) is necessary for (31) to hold.

The necessity of (31) for (33) is trivial. To show that (31) implies (33), note that the existence of $Y$ in (32) implies differentiability of $\mathbb{R}_{X}(d)$ [16], and therefore the maximum in (13) is attained by a unique $g(X)$ [16]. Since (31) establishes that $g(X)$ that attains the maximum in (13) is that in Table I, (33) follows.

Example. If $X$ is equiprobable on a finite group, (31) always holds. Indeed, in that case, equiprobable $Y$ satisfies (32).

Example. Gaussian source with mean-square error distortion satisfies the conditions of Theorem 3; indeed, $X=Y+Z$, where $X \sim \mathcal{N}\left(0, \sigma^{2} \mathbf{I}\right), Y \sim \mathcal{N}\left(0,\left(\sigma^{2}-d\right) \mathbf{I}\right) \Perp Z \sim \mathcal{N}(0, d \mathbf{I})$.

Theorem 3 presents a generalization of a result by Gerrish and Schultheiss [18], who showed that for the compression of a continuous random vector under the mean-square error distortion, the Shannon lower bound gives the actual value of rate-distortion function if and only if $X$ can be written as the sum of two independent random vectors $X=Y+Z$, where $Z \sim \mathcal{N}(0, d \mathbf{I})$.

Most continuous probability distributions do not meet the conditions of Theorem 3. In particular, an $X$ with indecomposable distribution cannot satisfy (32), regardless of the distortion measure. In contrast, as the following result shows, for finite alphabet sources Shannon's lower bound is always attained with equality, as long as target distortion $d$ is not too large.

Theorem 4. Let $X \in \mathcal{X}$, where $\mathcal{X}$ is a group of order $m$. Let the distortion measure satisfy (17), (25). Then, there exists a $d_{0}>0$ such that (31) and (33) hold for

$$
0 \leq \forall d \leq d_{0}
$$

Proof: Without loss of generality, we may assume that

$$
\mathcal{X}=\{0,1, \ldots, m-1\}
$$

and the group operation is modulo $m$ addition.

According to Theorem 3, we need to show the existence of such $Y$ that (32) holds. Toward this 
end, denote the $m \times m$ doubly stochastic circulant matrix

$$
A_{\lambda} \triangleq\left(\begin{array}{cccc}
P_{Z_{\lambda}}(0) & P_{Z_{\lambda}}(1) & \ldots & P_{Z_{\lambda}}(m-1) \\
P_{Z_{\lambda}}(m-1) & P_{Z_{\lambda}}(0) & \ldots & P_{Z_{\lambda}}(m-2) \\
\vdots & & \ddots & \vdots \\
P_{Z_{\lambda}}(1) & \ldots & P_{Z_{\lambda}}(m-1) & P_{Z_{\lambda}}(0)
\end{array}\right)
$$

Consider the system of linear equations

$$
\mathbf{q}^{T} A_{\lambda}=\mathbf{p}^{T}
$$

where $\mathbf{q}$ is an $m$-dimensional column vector, and $\mathbf{p}^{T}=\left(P_{X}(0), \ldots, P_{X}(m-1)\right)$. The existence of $Y$ in (32) is equivalent to requiring that (52) has solution q such that all entries of $\mathbf{q}$ are nonnegative; then, $P_{Y}=\mathbf{q}$ establishes (32) and therefore (31). Note that it is sufficient to ask that $\mathbf{q} \geq 0$ (elementwise) because the constraint $\mathbf{q}^{T} \mathbf{1}=1$, where $\mathbf{1}$ is an all-one vector, is satisfied automatically by any q satisfying (52): indeed, using that $A_{\lambda}$ is a stochastic matrix and $\mathbf{p}$ is a probability vector, we have

$$
\begin{aligned}
\mathbf{q}^{T} \mathbf{1} & =\mathbf{q}^{T} A_{\lambda} \mathbf{1} \\
& =\mathbf{p}^{T} \mathbf{1} \\
& =1
\end{aligned}
$$

To show the existence of a $d_{0}>0$ such that (52) has a solution with $\mathbf{q}=\mathbf{q}_{\lambda} \geq 0$ for all $d \leq d_{0}$, we use the following continuity argument. First, notice that

$$
\lim _{\lambda \rightarrow \infty} A_{\lambda}=\mathbf{I}
$$

Denote the smallest and the largest singular value of matrix $A$ by $\sigma_{\min }(A)$ and $\sigma_{\max }(A)$, respectively. Since the entries of $A_{\lambda}$ are continuous functions of $\lambda$, and the roots of a polynomial are continuous functions of its coefficients (e.g. [19, p. 363]),

$$
\forall \epsilon>0 \exists \lambda_{0}: \forall \lambda \geq \lambda_{0} \sigma_{\min }\left(A_{\lambda}\right) \geq 1-\epsilon
$$

A differentiation of the left side of (24) using (25) reveals that it is a decreasing function of $\lambda$ for large enough $\lambda$; therefore, $\forall \lambda \geq \lambda_{0}$ in (57) is equivalent to $\forall d \leq d_{0}$, where $d_{0}=\mathbb{E}\left[Z_{\lambda_{0}}\right]$.

Denote

$$
p_{\min } \triangleq \min _{m} P_{X}(m) .
$$


Noting that 1 is an eigenvector of $A_{\lambda}$, we write

$$
\begin{aligned}
\|\mathbf{q}-\mathbf{p}\|_{1} & =\left\|A_{\lambda}^{-1} \mathbf{p}^{T}-\mathbf{p}^{T}\right\|_{1} \\
& =\left\|A_{\lambda}^{-1}\left(\mathbf{p}-p_{\min } \mathbf{1}\right)^{T}-\left(\mathbf{p}-p_{\min } \mathbf{1}\right)^{T}\right\|_{1} \\
& \leq \max _{\mathbf{r}:\|\mathbf{r}\|_{1}=1-m p_{\min }}\left\|A_{\lambda}^{-1} \mathbf{r}^{T}-\mathbf{r}^{T}\right\|_{1} \\
& =\sigma_{\max }\left(A_{\lambda}^{-1}-\mathbf{I}\right)\left(1-m p_{\min }\right) \\
& \leq \frac{\epsilon\left(1-m p_{\min }\right)}{1-\epsilon}
\end{aligned}
$$

To ensure $\mathbf{q} \geq 0$, it suffices to consider $\epsilon=\frac{p_{\min }}{1-(m-1) p_{\min }}$, so that $\|\mathbf{q}-\mathbf{p}\|_{1} \leq p_{\min }$.

Remark 3. Denote the polynomial

$$
f(\nu) \triangleq \sum_{i=0}^{m-1} P_{Z_{\lambda}}(i) \nu^{i}
$$

Because $A_{\lambda}$ is a circulant matrix, its eigenvalues are simply $f\left(\omega^{0}\right), \ldots, f\left(\omega^{m-1}\right)$, where $\omega$ is $m$-th primitive root of unity (e.g. [20]). Moreover,

$$
F^{*} A_{\lambda} F=\operatorname{diag}\left(f\left(\omega^{0}\right), \ldots, f\left(\omega^{m-1}\right)\right)
$$

where $F$ is the unitary matrix whose $(i, j)$-th entry is equal to $\frac{1}{\sqrt{m}} \omega^{(i-1)(j-1)}$. In fact, $F$ corresponds to discrete Fourier transform, and thanks to the representation (65), a fast Fourier transform algorithm can be used to solve the system in (52) [21, Algorithm 4.8.1].

Example. For symbol error distortion

$$
\mathrm{d}(z)=1\{z=0\}
$$

(24) reduces to

$$
\frac{(m-1) \exp (-\lambda)}{1+(m-1) \exp (-\lambda)}=d
$$

which is equivalent to

$$
\lambda=\log (m-1)+\log \frac{1-d}{d} .
$$

Therefore,

$$
\begin{gathered}
Z_{\lambda}= \begin{cases}0 & \text { w. p. } 1-d \\
i & \text { w. p. } \frac{d}{m-1}, \quad i=1, \ldots, m-1\end{cases} \\
\mathbb{R}_{X}(d)=H(X)-h(d)-d \log (m-1) .
\end{gathered}
$$


Applying

$$
\sum_{i=0}^{m-1} \omega^{i k}=0, \quad 0<k<m,
$$

to (65), we conclude that the eigenvalues of $A_{\lambda}$ are 1 and $1-\frac{m}{m-1} d$ with multiplicity $m-1$. This implies that (62) is upper bounded by $p_{\min }$, and therefore, $\mathbb{R}_{X}(d)=\underline{\mathbb{R}}_{X}(d)$ according to Theorem 4 , as long as

$$
0 \leq d \leq \frac{m-1}{m} \frac{p_{\min }}{1-(m-1) p_{\min }}
$$

On the other hand, a direct computation of the solution to (52) using representation (65) reveals the well-known equality [22]

$$
\mathbb{R}_{X}(d)=\underline{\mathbb{R}}_{X}(d), \quad 0 \leq d \leq(m-1) P_{\min }
$$

The range of $d$ identified in (72) is smaller that in (73); the two ranges are equal if and only if $p_{\min }=\frac{1}{m}$, i.e. only for the equiprobable source. Thus, (62) gives a conservative estimate for the range in which the rate-distortion function is equal to Shannon's lower bound; the reason is that the solution to the equation (52) is in general not parallel to any eigenvector of $A_{\lambda}$. Of course, the exact value of $d_{0}$ in (49) can always be computed from the explicit solution to (52).

Since Theorem 4 ensures that the d-tilted information is given by (33) for a range of low distortions, one can apply (33) to (1) to conclude that for the compression of a discrete memoryless sources under a difference distortion measure,

$$
R(n, d, \epsilon)=\underline{R}(d)+\sqrt{\frac{\underline{\underline{V}}}{n}} Q^{-1}(\epsilon)+O\left(\frac{\log n}{n}\right),
$$

for all $d \leq d_{0}$.

Unfortunately, continuous sources rarely meet the conditions of Theorem 3, and thus (74) does not hold in general. Nevertheless, as we will see, lattice quantization of continuous sources often approaches (74). This striking phenomenon is the major focus of the remainder of the paper. The next section introduces the topic by discussing lattice coverings of space.

\section{LATTICE QUANTIZER ENTROPY}

The purpose of this section is to bound the output entropy of a lattice quantizer in terms of the differential entropy of the source and the size of the lattice cell. Let $\mathcal{C}$ be a non-degenerate lattice in $\mathbb{R}^{n}$ :

$$
\mathcal{C} \triangleq\left\{c=G \cdot i: i \in \mathbb{Z}^{n}\right\}
$$


where the $n \times n$ generator matrix $G$ is non-singular.

The nearest-neighbor $\mathcal{C}$-quantizer is the mapping $q_{\mathcal{C}}: \mathbb{R}^{n} \mapsto \mathcal{C}$ defined by ${ }^{3}$

$$
q_{\mathcal{C}}(x) \triangleq \underset{c \in \mathcal{C}}{\operatorname{argmin}}\|x-c\|
$$

and the Voronoi cell $\mathcal{V}_{\mathcal{C}}(c)$ is the set of all points quantized to $c$ :

$$
\mathcal{V}_{\mathcal{C}}(c) \triangleq\left\{x \in \mathbb{R}^{n}: q_{\mathcal{C}}(x)=c\right\}
$$

The ties in (76) are resolved so that the resulting Voronoi cells are congruent. We denote by $V_{\mathcal{C}}$ the cell volume of lattice $\mathcal{C}$ :

$$
\begin{aligned}
V_{\mathcal{C}} & \triangleq \operatorname{Vol}\left(\mathcal{V}_{\mathcal{C}}(0)\right) \\
& =|\operatorname{det} G|
\end{aligned}
$$

In the sequel we will be concerned with properties of lattices in the limit of increasing point density, or vanishing cell volume. As evident from (75), a scaling of $G$ by $\frac{V^{\frac{1}{n}}}{|\operatorname{det} G|^{\frac{1}{n}}}$ results in the lattice of cell volume $V$. Fixing $G$ and considering lattices generated by $\frac{V^{\frac{1}{n}}}{|\operatorname{det} G|^{\frac{1}{n}}}$, we obtain a continuum of lattices parameterized by $V$. We will be interested in the rate-distortion behavior of lattice coverings as $V \rightarrow 0$.

For lattice $\mathcal{C} \in \mathbb{R}^{n}$, denote the lattice-quantized version of $n$-dimensional random variable $X$ by

$$
X_{\mathcal{C}} \triangleq q_{\mathcal{C}}(X)+U_{\mathcal{C}}
$$

where random variable $U_{\mathcal{C}}$ is uniform on $\mathcal{V}_{\mathcal{C}}(0)$. See Fig. 1 . Clearly, as the quantization cells become smaller, the distribution of $X_{\mathcal{C}}$ becomes a better approximation of the distribution of $X$. The next result, essentially contained in [23], formalizes this intuition by underlining the connection between the entropy $X_{\mathcal{C}}$ and the differential entropy of $X$.

Theorem 5. Let $X$ be a random variable and let $\mathcal{C}$ be a lattice in $\mathbb{R}^{n}$. Then,

$$
H\left(q_{\mathcal{C}}(X)\right)=h\left(X_{\mathcal{C}}\right)+\log \frac{1}{V_{\mathcal{C}}} .
$$

Furthermore, if 4

$$
H(\lfloor X\rfloor)<\infty
$$

\footnotetext{
${ }^{3}$ Here, $\|\cdot\|$ is the Euclidean norm. As we will see in Section VI, most of the results can be generalized to other norms.

${ }^{4}$ For vector $x^{n},\left\lfloor x^{n}\right\rfloor$ denotes the vector of integer parts of its components, that is, $\left\lfloor x^{n}\right\rfloor=\left(\left\lfloor x_{1}\right\rfloor, \ldots,\left\lfloor x_{n}\right\rfloor\right)$.
} 


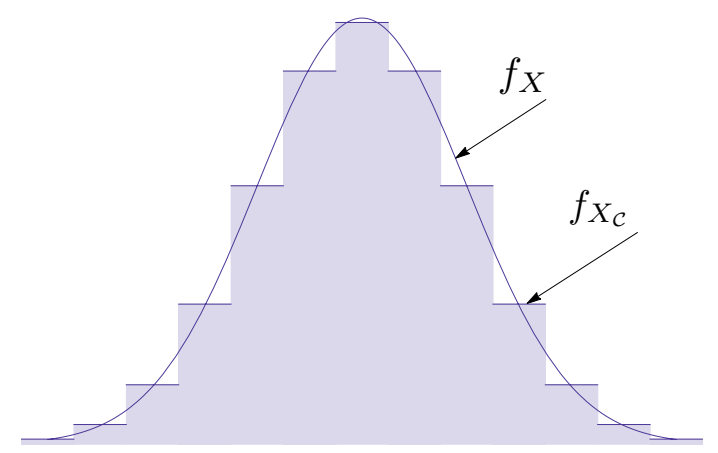

Fig. 1: Example: densities of $X$ and $X_{\mathcal{C}}$ for $n=1$ and $\mathcal{C}$ the integer lattice.

then for any sequence of lattices with vanishing cell volume,

$$
\lim _{V_{\mathcal{C}} \rightarrow 0} h\left(X_{\mathcal{C}}\right)=h(X)
$$

Proof: Note that $X_{\mathcal{C}}$ has a density even if $X$ does not. The distribution of discrete random variable $q_{\mathcal{C}}(X)$ satisfies, for any $c \in \mathcal{C}$,

$$
\frac{1}{V_{\mathcal{C}}} P_{q_{\mathcal{C}}(X)}(c)=f_{X_{\mathcal{C}}}\left(c-u_{0}\right), \quad \forall u_{0} \in \mathcal{V}_{\mathcal{C}}(0)
$$

Taking logarithms of both sides of (84) and then an expectation with respect to $f_{X_{\mathcal{C}}}$ reveals (81).

To show (83), continue (84) as

$$
f_{X_{\mathcal{C}}}\left(c-u_{0}\right)=\mathbb{E}\left[f_{X}\left(c-U_{\mathcal{C}}\right)\right]
$$

By Jensen's inequality

$$
h(X) \leq h\left(X_{\mathcal{C}}\right)
$$

If $h(X)=+\infty$, due to (86) there is nothing to prove. For $h(X)<+\infty$, the validity of (83) under assumption (82) is shown in [23].

Theorem 5 holds even if $X$ does not have a density; in that case, $h(X)=-\infty$. Theorem 5 also holds for the more general case of non-lattice partitions of $\mathbb{R}^{n}$ into sets of equal volume. Prior to Csiszár, the validity of (83) under a more restrictive assumption was proved by Rényi [24, Theorem $4]$. 
Remark 4. If $h(X)>-\infty$, using (85) it is easy to verify that

$$
D\left(X \| X_{\mathcal{C}}\right)=-h(X)+h\left(X_{\mathcal{C}}\right)
$$

so (81) can be rewritten as

$$
H\left(q_{\mathcal{C}}(X)\right)=h(X)+\log \frac{1}{V_{\mathcal{C}}}+D\left(X \| X_{\mathcal{C}}\right)
$$

and (83) as

$$
\lim _{V_{\mathcal{C}} \rightarrow 0} D\left(X \| X_{\mathcal{C}}\right)=0
$$

Remark 5. Assumption (82) is needed to ensure that the tails of $f_{X}(x) \log f_{X}(x)$ are well behaved. If $f_{X}$ is supported on a compact, then (83) holds for all continuous $f_{X}$. Indeed, applying the mean value theorem to (85), for each $c \in \mathcal{C}$ we note the existence of $u_{c} \in \mathcal{V}_{\mathcal{C}}(0)$ such that

$$
f_{X}\left(c-u_{c}\right)=f_{X_{c}}\left(c-u_{0}\right), \quad \forall u_{0} \in \mathcal{V}_{\mathcal{C}}(0)
$$

It follows that $h\left(X_{\mathcal{C}}\right)$ is the Riemann sum for $f_{X}(x) \log \frac{1}{f_{X}(x)}$ and the partition generated by Voronoi cells of $\mathcal{C}$ labeled by $c-u_{c}, c \in \mathcal{C}$, that is,

$$
\begin{aligned}
h\left(X_{\mathcal{C}}\right) & =\sum_{c \in \mathcal{C}} V_{\mathcal{C}} f_{X_{\mathcal{C}}}(c) \log \frac{1}{f_{X_{\mathcal{C}}}(c)} \\
& =\sum_{c \in \mathcal{C}} V_{\mathcal{C}} f_{X}\left(c-u_{c}\right) \log \frac{1}{f_{X}\left(c-u_{c}\right)}
\end{aligned}
$$

Convergence to $h(X)$ follows by the definition of Riemann integral.

Remark 6. Csiszár [23] showed the validity of (83) under the following more general than (82) assumption. Suppose there exists some Borel measurable partition $\left\{\mathcal{B}_{1}, \mathcal{B}_{2}, \ldots\right\}$ of $\mathbb{R}^{n}$ into sets of finite Lebesgue measure such that the following two conditions are satisfied.

1)

$$
\sum_{i} P_{X}\left(\mathcal{B}_{i}\right) \log \frac{1}{P_{X}\left(\mathcal{B}_{i}\right)}<\infty
$$

2) There exist $\rho>0$ and $s \in \mathbb{N}$ such that for all $k$, the distance between $\mathcal{B}_{k}$ and $\mathcal{B}_{\ell}, k \neq \ell$, is greater than $\rho$ for all but at most $s$ indices $\ell$.

Recently, Koch [25] showed that the rate-distortion function (for the mean squared error) is infinite for all $d>0$ if $H(\lfloor X\rfloor)=\infty$. Thus, assumption (82) is as general as Csiszár's assumption in most cases of interest. 
Remark 7. The following considerations help to check whether a given random vector satisfies (82). As shown in Appendix A,

$$
H(\lfloor X\rfloor)<\mathbb{E}\left[\log R_{n}\left(\|\lfloor X\rfloor\|^{2}\right)\right]+\log e+\log \left(\mathbb{E}\left[\log R_{n}\left(\|\lfloor X\rfloor\|^{2}\right)\right]+1\right),
$$

where $R_{n}\left(\sigma^{2}\right)$ is the number of integer-valued $n$-vectors whose Euclidean norm does not exceed $\sigma$. In particular, for $n=1, R_{1}(k)=1+2 k$, so

$$
\mathbb{E}\left[\log R_{1}\left(\|X\|^{2}\right)\right]=\mathbb{E}[\log (2|\lfloor X\rfloor|+1)]
$$

More generally, for $n \geq 1$ (Appendix A),

$\mathbb{E}\left[\log R_{n}\left(\|\lfloor X\rfloor\|^{2}\right)\right] \leq n \mathbb{E}\left[\log \left(1+\frac{1}{\sqrt{n}}\|X\|\right)\right]+n \log \sqrt{2 \pi e}-\frac{1}{2} \log (n+2)-\log \sqrt{\pi}+\frac{2}{n+2} \log e$.

Bounds (94) and (96) imply that a sufficient condition for (82) is $\mathbb{E}\left[\log \left(1+\frac{1}{\sqrt{n}}\|X\|\right)\right]<\infty$. This in turn holds for any source vector for which $\mathbb{E}\left[\|X\|^{\alpha}\right]<\infty$ for some $\alpha>0 .^{5}$

The strength of Theorem 5 is that it requires only a very mild assumption on the source density, namely, (82). The weakness is that it does not offer any estimate on the speed of convergence to the limit in (83) (or, equivalently, in (89)); such an estimate will be crucial in our study of the behavior of the output entropy of lattice quantizers in the limit of increasing dimension. Naturally, for the relative entropy $D\left(X \| X_{\mathcal{C}}\right)$ to be small, the probability density function of $X$ should not change too abruptly within a single quantization cell.

The following smoothness condition will be instrumental.

Definition 1 ( $v$-regular density). Let $v: \mathbb{R}^{n} \mapsto \mathbb{R}^{+}$. Differentiable probability density function $f_{X}$ is called v-regular if

$$
\left\|\nabla f_{X}(x)\right\| \leq v(x) f_{X}(x), \quad \forall x \in \mathbb{R}^{n}
$$

Remark 8. Note that differentiable $f_{X}$ has finite variation if and only if (97) holds with equality for $f_{X}$-a.s. $x$ and some function $v$ such that $\mathbb{E}[v(X)]<\infty$; the total variation of $f_{X}$ is given by $\mathbb{E}[v(X)]$. Remark 9. If $f_{X^{n}}$ is a product distribution and $f_{X_{i}}$ is $v_{i}$-regular, then $f_{X^{n}}$ is $v$-regular, where

$$
v\left(x^{n}\right)=\left\|v_{1}\left(x_{1}\right), \ldots, v_{n}\left(x_{n}\right)\right\|
$$

\footnotetext{
${ }^{5}$ These sufficient conditions were pointed out in [25] without proof.
} 
Definition 1 presents a slight generalization of a smoothness condition recently suggested by Polyanskiy and $\mathrm{Wu}$ [26]. Namely, they considered densities satisfying

$$
\left\|\nabla \log f_{X}(x)\right\| \leq c_{1}\|x\|+c_{0}, \quad \forall x \in \mathbb{R}^{n}
$$

for some $c_{0} \geq 0, c_{1}>0$. Clearly, if $f_{X}$ satisfies (99), then it is also $v$-regular with $v(x)=c_{1}\|x\|+c_{0}$; and vice versa, if $f_{X}$ is never zero and satisfies (97) with

$$
v(x)=c_{1}\|x\|+c_{0},
$$

then it also satisfies (99). A wide class of densities satisfying (99) is identified in [26]. In particular, the density of $B+Z$, with $B \Perp Z$ and $Z \sim \mathcal{N}\left(0, \sigma^{2} \mathbf{I}\right)$ satisfies (99) with $c_{0}=\frac{4 \log e}{\sigma^{2}} \mathbb{E}[\|B\|], c_{1}=\frac{2 \log e}{\sigma^{2}}$. Likewise, if the density of $Z$ satisfies (99) with $c_{0}=c_{0}^{\prime}, c_{1}=c_{1}^{\prime}$, then that of $B+Z$, where $\|B\| \leq b$ a.s., $B \Perp Z$, satisfies (99) with $c_{1}=c_{1}^{\prime}, c_{0}=c_{0}^{\prime}+c_{1}^{\prime} b$.

Before we present a bound on $D\left(X \| X_{\mathcal{C}}\right)$ for $X$ with regular probability density function, we introduce the following notation. Denote by $\mathcal{B}_{r}$ an $n$-dimensional ball of radius $r$ centered at zero:

$$
\mathcal{B}_{r} \triangleq\left\{x \in \mathbb{R}^{n}:\|x\| \leq r\right\} .
$$

The covering radius of lattice $\mathcal{C}$ is the minimum $r$ such that the set $\mathcal{C}+\mathcal{B}_{r}$, composed of balls centered at lattice points, covers $\mathbb{R}^{n}$ :

$$
r_{\mathcal{C}} \triangleq \min \left\{r: \mathcal{C}+\mathcal{B}_{r} \supseteq \mathbb{R}^{n}\right\}
$$

Proposition 1. Assume that $X \in \mathbb{R}^{n}$ has v-regular density. The relative entropy between $X$ and $X_{\mathcal{C}}$ is bounded as

$$
D\left(X \| X_{\mathcal{C}}\right) \leq 2 r_{\mathcal{C}} \mathbb{E}\left[v_{\mathcal{C}}(X)\right]
$$

where we denoted for brevity

$$
v_{\mathcal{C}}(x)=\max _{u \in \mathcal{V}_{\mathcal{C}}\left(q_{\mathcal{C}}(x)\right)} v(u)
$$

Proof: Observe that,

$$
\begin{aligned}
& \left|\log f_{X}(a)-\log f_{X}(b)\right| \\
= & \left|\int_{0}^{1}\left(\nabla \log f_{X}(t a+(1-t) b), a-b\right) d t\right| \\
\leq & \|a-b\| \log e \int_{0}^{1} v(t a+(1-t) b) d t \\
\leq & \max _{0 \leq t \leq 1} v(t a+(1-t) b)\|a-b\| \log e
\end{aligned}
$$


where (106) holds by Cauchy-Schwarz inequality. Using (90) and (107), we evaluate the relative entropy between $X$ and $X_{\mathcal{C}}$ as

$$
\begin{aligned}
& D\left(X \| X_{\mathcal{C}}\right) \\
= & \sum_{c \in \mathcal{C}} \int_{\mathcal{V}_{\mathcal{C}}(0)} f_{X}(c-u) \log \frac{f_{X}(c-u)}{f_{X}\left(c-u_{c}\right)} d u \\
\leq & 2 r_{\mathcal{C}} \log e \sum_{c \in \mathcal{C}} \int_{\mathcal{V}_{\mathcal{C}}(0)} f_{X}(c-u) \max _{\xi \in \mathcal{V}_{\mathcal{C}}(0)} v(c-u+\xi) d u \\
= & 2 r_{\mathcal{C}} \mathbb{E}\left[v_{\mathcal{C}}(X)\right] \log e,
\end{aligned}
$$

where $u_{c}$ is defined in (90).

Remark 10. If $v$ is convex, (103) can be strenghtened to

$$
D\left(X \| X_{\mathcal{C}}\right) \leq r_{\mathcal{C}} \log e\left(\mathbb{E}[v(X)]+\mathbb{E}\left[v_{\mathcal{C}}(X)\right]\right)
$$

Indeed, applying Jensen's inequality to (106) results in

$$
\left|\log f_{X}(a)-\log f_{X}(b)\right| \leq \frac{\log e}{2}(v(a)+v(b))\|a-b\|
$$

and modifying (109) accordingly results in (111). In particular, for $v(x)$ in (100) we obtain from (111):

$$
\begin{aligned}
& \left|\log f_{X}(a)-\log f_{X}(b)\right| \\
\leq & \frac{\log e}{2}\|a-b\|\left(c_{1}\|a\|+c_{1}\|b\|+2 c_{0}\right),
\end{aligned}
$$

which is the result of [26].

Next, we consider the possibilities of lattice quantization in large dimension $n$.

\section{LATTICE RATE-DISTORTION FUNCTION}

Lattice coverings of space become more efficient as the dimension increases. In this section we study the minimum entropy attainable by lattice quantizers in the limit of large $n$.

Covering efficiency of lattice $\mathcal{C}$ is measured by

$$
\begin{aligned}
\rho_{\mathcal{C}} & \triangleq\left(\frac{\operatorname{Vol}\left(\mathcal{B}_{r_{\mathcal{C}}}\right)}{V_{\mathcal{C}}}\right)^{\frac{1}{n}} \\
& =r_{\mathcal{C}} \frac{b_{n}^{\frac{1}{n}}}{V_{\mathcal{C}}^{\frac{1}{n}}}
\end{aligned}
$$


where $b_{n}$ is the volume of a unit ball:

$$
b_{n} \triangleq \frac{\pi^{\frac{n}{2}}}{\Gamma\left(\frac{n}{2}+1\right)}
$$

By definition,

$$
\rho_{\mathcal{C}} \geq 1
$$

and the closer $\rho_{\mathcal{C}}$ is to 1 the more sphere-like the Voronoi cells of $\mathcal{C}$ are and the better lattice $\mathcal{C}$ is for covering.

For distortion measure $d: \mathbb{R}^{n} \times \mathbb{R}^{n} \mapsto \mathbb{R}^{+}$, denote minimum output entropy attainable by lattice quantization of the random vector $X \in \mathbb{R}^{n}$ by

$$
\mathbb{L}_{X}(d) \triangleq \inf _{\mathcal{C}: \mathrm{d}\left(X, q_{\mathcal{C}}(X)\right) \leq d \text { a.s. }} H\left(q_{\mathcal{C}}(X)\right),
$$

The definition in (118) parallels the definition of $\epsilon$-entropy [27]:

$$
\mathbb{H}_{X^{n}}(d) \triangleq \inf _{\substack{q: \mathbb{R}^{n} \mapsto \mathbb{R}^{n} \\ \mathrm{~d}\left(X^{n}, q\left(X^{n}\right)\right) \leq d \text { a.s. }}} H\left(q\left(X^{n}\right)\right),
$$

with the difference that in (119) the infimization is performed over all mappings $q: \mathbb{R}^{n} \mapsto \mathbb{R}^{n}$ and not just lattice quantizers. For that reason, we call the function in (118) lattice $\epsilon$-entropy.

The next result characterizes the lattice $\epsilon$-entropy of an $n$-dimensional random variable.

Proposition 2. Consider lattice quantization of a continuous random vector $X^{n}$ with $h\left(X^{n}\right)>-\infty$ under the mean-square error distortion (20). Lattice e-entropy is characterized as

$$
\begin{aligned}
\mathbb{L}_{X^{n}}(d) & =h\left(X^{n}\right)+\frac{n}{2} \log \frac{1}{n d}-\log b_{n} \\
& +\inf _{\mathcal{C}: r_{\mathcal{C}} \leq \sqrt{n d}}\left\{D\left(X^{n} \| X_{\mathcal{C}}^{n}\right)+n \log \rho_{\mathcal{C}}\right\},
\end{aligned}
$$

where $X_{\mathcal{C}}, \rho_{\mathcal{C}}$ and $b_{n}$ are defined in (80), (114) and (116), respectively.

Proof: We use (88) and (115) to expand

$$
H\left(q_{\mathcal{C}}\left(X^{n}\right)\right)=h\left(X^{n}\right)+n \log \frac{1}{r_{\mathcal{C}}}-\log b_{n}+D\left(X \| X_{\mathcal{C}}^{n}\right)+n \log \rho_{\mathcal{C}}
$$

and (120) is immediate upon applying (114) and

$$
d=\frac{r_{\mathcal{C}}^{2}}{n}
$$


Since the term inside the infimum in (120) is nonnegative, $\mathbb{L}_{X^{n}}(d)$ is lower-bounded by the first three terms in (120). In fact, as we will see below in Theorem 7, for small $d, \mathbb{L}_{X^{n}}(d)$ is well approximated by those three terms.

Definition 2. Lattice rate-distortion function for the compression of a sequence of random variables $X_{1}, X_{2}, \ldots$ is defined by

$$
L(d) \triangleq \limsup _{n \rightarrow \infty} \frac{1}{n} \mathbb{L}_{X^{n}}(d)
$$

The operational rate-distortion function can be defined as follows.

Definition 3. Rate-distortion function for the compression of a sequence of random variables $X_{1}, X_{2}, \ldots$ is defined by

$$
R(d) \triangleq \limsup _{n \rightarrow \infty} \frac{1}{n} \mathbb{H}_{X^{n}}(d)
$$

where $\mathbb{H}_{X^{n}}(d)$ is the $\epsilon$-entropy of $X^{n}$ defined in (119).

Note that

$$
\mathbb{R}_{X^{n}}(d) \leq \mathbb{H}_{X^{n}}(d)
$$

where $\mathbb{R}_{X^{n}}(d)$ is the $n$-dimensional minimal mutual information quantity defined in (10) (replacing $\mathrm{X}$ by $\left.X^{n}\right)$.

A variable-length code for random variable $X \in \mathbb{R}^{n}$ is a pair of mappings $f: \mathbb{R}^{n} \mapsto\{0,1\}^{\star}$ and $\mathrm{g}:\{0,1\}^{\star} \mapsto \mathbb{R}^{n}$, where $\{0,1\}^{\star}$ is the set of all binary strings, including the empty string. ${ }^{6}$ The operational characteristic of code $(f, g)$ is the tradeoff between the maximal distortion esssup $\mathrm{d}(X, \mathrm{~g}(\mathrm{f}(X)))$ and the encoded length it achieves. Since the encoded length is a random variable, the encoded length is measured in some stochastic sense. A popular criterion is the average length, given by $\mathbb{E}[\ell(f(X))]$, where $\ell(\cdot)$ is the length of the binary string in its argument. Another criterion is the excess encoded length compatible with excess probability $\epsilon$, given by the maximum integer $k$ such that $\mathbb{P}[\ell(f(X))>k] \leq \epsilon$. The rate of the code is simply the length (either excess or average) normalized by the blocklength.

The operational meaning of (123) is the asymptotically minimum average rate compatible with maximal distortion $d$. Indeed, it is well known that $L_{S}^{\star}$, the minimum average length required to

\footnotetext{
${ }^{6}$ If the alphabet is nonbinary, the base of the logarithms throughout is adjusted accordingly.
} 
losslessly encode discrete random variable $S$ is bounded in terms of the entropy of $S$ as [28], [29]

$$
\begin{aligned}
H(S)-\log _{2}(H(S)+1)-\log _{2} e & \leq L_{S}^{\star} \\
& \leq H(S)
\end{aligned}
$$

Substituting $S=q_{\mathcal{C}}\left(X^{n}\right)$ and dividing through by $n$, we conclude that (123) is equivalent to

$$
L(d)=\limsup _{n \rightarrow \infty} \frac{1}{n} \inf _{\mathcal{C}: \mathrm{d}\left(X^{n}, q_{\mathcal{C}}\left(X^{n}\right)\right) \leq d \text { a.s. }} L_{q_{\mathcal{C}}\left(X^{n}\right)}^{\star} .
$$

The following result demonstrates the existence of covering-efficient lattices.

Theorem 6 (Rogers [9, Theorem 5.9]). For each $n \geq 3$, there exists an $n$-dimensional lattice $\mathcal{C}_{n}$ and constant $c$ with covering efficiency

$$
n \log \rho_{\mathcal{C}_{n}} \leq \log _{2} \sqrt{2 \pi e}(\log n+\log \log n+c) .
$$

The main result of this section is the following.

Theorem 7. Assume that for some integer $m \geq 0$ and scalar $\mu \geq 1$, the ergodic process $X_{1}, X_{2}, \ldots$ is such that:

(i) The density $f_{X^{n}}$ is $v_{n}$-regular with

$$
v_{n}\left(x^{n}\right)=\sqrt{n} \vee\left(\frac{\left\|x^{n}\right\|}{\sqrt{n}}\right)
$$

where $\mathrm{v}: \mathbb{R}_{+} \mapsto \mathbb{R}_{+}$is convex and nondecreasing.

(ii) There exists $\mu<\infty$ such that

$$
\mathbb{E}\left[\mathrm{\vee}\left(i \frac{\left\|x^{n}\right\|}{\sqrt{n}}\right)\right] \leq i \mu, \quad i=1,2 ; n=1,2, \ldots .
$$

Then, as $d \rightarrow 0$, the lattice rate-distortion function under the mean-square error distortion expands as,

$$
L(d)=h+\frac{1}{2} \log \frac{1}{d}-\log \sqrt{2 \pi e}+O(\sqrt{d})
$$

where

$$
h \triangleq \limsup _{n \rightarrow \infty} \frac{1}{n} h\left(X^{n}\right)
$$


Proof: Note, using Stirling's approximation,

$$
\log b_{n}=\frac{n}{2} \log \frac{2 \pi e}{n}-\frac{1}{2} \log n+O(1) .
$$

Substituting (134) into (120) and using the fact that the expression under the infimum in (120) is nonnegative, we conclude that as $n \rightarrow \infty$,

$$
\mathbb{L}_{X^{n}}(d) \geq h\left(X^{n}\right)+\frac{n}{2} \log \frac{1}{d}-n \log \sqrt{2 \pi e}+\frac{1}{2} \log n+O(1) .
$$

For the upper bound, we relax the infimum in (120) by letting $\mathcal{C}_{n}$ be the sequence of lattices that has covering radius in (122) and that achieves the Rogers bound (129):

$$
\begin{aligned}
\mathbb{L}_{X^{n}}(d) & \leq h\left(X^{n}\right)+\frac{n}{2} \log \frac{1}{d}-n \log \sqrt{2 \pi e} \\
& +D\left(X^{n} \| X_{\mathcal{C}_{n}}^{n}\right)+O(\log n) .
\end{aligned}
$$

To bound $D\left(X^{n} \| X_{\mathcal{C}}^{n}\right)$, consider $v$-regular $f_{X}$ with $v(x)=v(\|x\|)$ that is convex and nondecreasing. Using (112), we observe that for all $a$ and $b$ from the same lattice cell,

$$
\begin{aligned}
\left|\log f_{X}(a)-\log f_{X}(b)\right| & \leq \frac{\log e}{2}(v(\|a\|)+v(\|b\|))\|a-b\| \\
& \leq \frac{\log e}{2}\left(v(\|a\|)+\frac{1}{2} v(2\|a\|)+\frac{1}{2} v(2\|a-b\|)\right)\|a-b\| \\
& \leq \log e\left(v(\|a\|)+\frac{1}{2} v(2\|a\|)+\frac{1}{2} v(4 \sqrt{n d})\right) \sqrt{n d}
\end{aligned}
$$

where (138) is by the triangle inequality and the convexity of $v$, and (139) holds because $\|a-b\| \leq 2 r_{\mathcal{C}}$ and $v$ is nondecreasing. Replacing $a$ and $b$ by $X$ and $X_{\mathcal{C}}$, taking an expectation of (139) and applying (130) and (131), we conclude

$$
D\left(X^{n} \| X_{\mathcal{C}}^{n}\right) \leq \log e\left(2 \mu+\frac{1}{2} \mathrm{v}(4 \sqrt{d})\right) n \sqrt{d} .
$$

Combining (140) and (136) leads to (132).

Remark 11. An important special case satisfying the conditions of Theorem 7 is the following. Suppose that

$$
\mathbb{E}\left[\left\|X^{n}\right\|\right] \leq \sqrt{n} \mu, \quad n=1,2, \ldots,
$$

and $f_{X^{n}}$ is $\sqrt{n} c_{0}+c_{1}\|x\|$-regular. Then, in particular, (140) becomes,

$$
D\left(X^{n} \| X_{\mathcal{C}}^{n}\right) \leq 2 n \sqrt{d} \log e\left(c_{1} \mu+c_{0}+\sqrt{d}\right) .
$$


Remark 12. For fixed $n$ and any random vector $X^{n}$ with $h\left(X^{n}\right)>-\infty$, as $d \rightarrow 0$ we have the expansion

$$
\mathbb{L}_{X^{n}}(d)=h\left(X^{n}\right)+\frac{n}{2} \log \frac{1}{d}-\log b_{n}+n \log \min _{\mathcal{C}} \rho_{\mathcal{C}}+o(1),
$$

which is obtained by applying (89) to (120). For regular densities, applying (140) to (120) yields (143) with $o(1)$ replaced by $n O(\sqrt{d})$. The thinnest lattice covering is proven in dimensions 1 to 5 to be $A_{n}^{*}$ (Voronoi's principal lattice of the first type) [30], which has covering efficiency

$$
\rho_{A_{n}^{*}}=b_{n}^{\frac{1}{n}}(n+1)^{\frac{1}{2 n}} \sqrt{\frac{n(n+2)}{12(n+1)}},
$$

so for $n=1,2, \ldots, 5$,

$$
\mathbb{L}_{X^{n}}(d)=h\left(X^{n}\right)+\frac{n}{2} \log \frac{1}{d}+\frac{n}{2} \log \frac{n(n+2)}{12(n+1)}+\frac{1}{2} \log (n+1)+o(1) .
$$

Actually, $A_{n}^{*}$ is the thinnest lattice covering known in all dimensions $n \leq 23$. But $A_{24}^{*}$ has covering efficiency $1.189 \ldots$ and is inferior to the Leech lattice $\Lambda_{24}$, for which $\rho_{\Lambda_{24}}=1.090 \ldots$

We are now in the position to compare the performance of lattice quantization to the optimum performance theoretically attainable. Denote

$$
\underline{R}(d) \triangleq \limsup _{n \rightarrow \infty} \frac{1}{n} \mathbb{R}_{X^{n}}(d),
$$

where the $n$-dimensional Shannon lower bound $\mathbb{R}_{X^{n}}(d)$ is defined in (19).

The following corollary summarizes the main message of Theorem 7.

Corollary 1. Assume that the process $X_{1}, X_{2}, \ldots$ satisfies the regularity conditions of Theorem 7. Then, as $d \rightarrow 0$, Shannon's lower bound to rate-distortion function under the mean-square error distortion is approached by lattice quantization:

$$
L(d)=\underline{R}(d)+O(\sqrt{d}) .
$$

Proof: Shannon's lower bound in (18) and (23) implies via (125) that

$$
R(d) \geq \underline{R}(d)=h+\frac{1}{2} \log \frac{1}{d}-\log \sqrt{2 \pi e},
$$

and, since $L(d) \geq R(d)$, (206) follows by comparing to (132).

Remark 13. Applying (129) and (134) to (143), we conclude

$$
\lim _{d \rightarrow 0}\left[\mathbb{L}_{X^{n}}(d)-\frac{1}{2} \log \frac{1}{d}\right]=h\left(X^{n}\right)-n \log \sqrt{2 \pi e}+O(\log n),
$$


and therefore

$$
\limsup _{n \rightarrow \infty} \frac{1}{n} \lim _{d \rightarrow 0}\left[\mathbb{L}_{X^{n}}(d)-\frac{1}{2} \log \frac{1}{d}\right]=h-\log \sqrt{2 \pi e} .
$$

In comparison, (132) leads to

$$
\lim _{d \rightarrow 0} \limsup _{n \rightarrow \infty} \frac{1}{n}\left[\mathbb{L}_{X^{n}}(d)-\frac{1}{2} \log \frac{1}{d}\right]=h-\log \sqrt{2 \pi e} .
$$

We stress that in contrast to (151), which demands regularity conditions on $f_{X^{n}}$ to ensure that $D\left(X^{n} \| X_{\mathcal{C}}^{n}\right)$ vanishes fast enough with vanishing cell volume even as the dimension increases, the result in (150) requires virtually no assumptions on the distribution of $X^{n}$. While the result in (151) is new, statements similar to (150) are found in the existing literature. The counterpart of (150) for dithered lattice quantization is contained in [31]. The following result was shown by Linkov [5] and revisited by Linder and Zamir [6]:

$$
\lim _{d \rightarrow 0}\left[\mathbb{R}_{X^{n}}(d)-\frac{1}{2} \log \frac{1}{d}\right]=h\left(X^{n}\right)-n \log \sqrt{2 \pi e},
$$

where $\mathbb{R}_{X^{n}}(d)$ is the $n$-dimensional minimal mutual information quantity defined in (10) (replacing $X$ by $X^{n}$ ). The result in (152) does not imply the existence of an $n$-dimensional quantizer whose rate satisfies (152). Moreover, Koch and Vazquez-Vilar [32] recently showed that if one replaces $\mathbb{R}_{X^{n}}(d)$ in (152) by the minimum output entropy attainable by an $n$-dimensional quantizer operating at average distortion $d$, then the resulting limit as $d \rightarrow 0$ is strictly greater than the right side of (152).

Nevertheless, (152) does have an operational meaning in terms of dithered quantization. The proofs in [5], [6] start with

$$
\mathbb{R}_{X}(d) \leq I(X ; X+Z)
$$

where the choice of $Z$ satisfies $\mathbb{E}[\mathrm{d}(X, X+Z)] \leq d$. Since operationally, $I(X ; X+Z)$ corresponds to the quantization rate (see e.g. [31]) of $X$ dithered by $Z$, (152) can be reinterpreted in terms of the minimum rate of dithered quantization.

Remark 14. If, instead of requiring that $\mathbb{P}\left[\frac{1}{n}\left\|X^{n}-q_{\mathcal{C}}\left(X^{n}\right)\right\|^{2} \leq d\right]=1$ as in (118), we ask only that $\mathbb{E}\left[\frac{1}{n}\left\|X^{n}-q_{\mathcal{C}}\left(X^{n}\right)\right\|^{2}\right] \leq d$, then the analog of the result in (149) can be alternatively obtained as follows. Denote the minimum of normalized second moments over all $n$-dimensional lattices by

$$
G_{n}^{\star} \triangleq \min _{\mathcal{C}_{n}} \frac{\mathbb{E}\left[\left\|U_{\mathcal{C}_{n}}\right\|\right]}{n V_{\mathcal{C}_{n}}^{\frac{2}{n}}},
$$

where $U_{\mathcal{C}_{n}}$ is uniform on $\mathcal{V}_{\mathcal{C}_{n}}(0)$.In [33, (25)], it is shown that $G_{n}^{\star}$ converges to $1 /(2 \pi e)$ at a rate

$$
\frac{1}{n} \log \left(2 \pi e G_{n}^{\star}\right)=O\left(\frac{\log n}{n}\right),
$$


a result which Zamir and Feder attributed to Poltyrev. Let $\mathcal{C}_{n, d}^{\star}$ be the lattice whose normalized second moment equals $G_{n}^{\star}$ rescaled so that its mean square error (with respect to $X^{n}$ ) is $d$. Using $\mathcal{C}_{n, d}^{\star}$ in [12, Theorem 1], one concludes that

$$
\lim _{d \rightarrow 0} \frac{1}{n}\left(H\left(q_{\mathcal{C}_{n, d}^{\star}}\left(X^{n}\right)\right)+\frac{1}{2} \log d\right)=\frac{1}{n}\left(h\left(X^{n}\right)+\frac{1}{2} \log \left(G_{n}^{\star}\right)\right) .
$$

Substituting (155) into (156), one obtains the same asymptotics as in (149). A gentle modification of the above argument (apparent from [33, (26)] and [12, Lemma 1]) leads to (149) for the maximal distortion criterion as well.

Remark 15. The limit in (150) can be re-written as

$$
\lim _{d \rightarrow 0} \frac{\mathbb{L}_{X^{n}}(d)}{\frac{n}{2} \log \frac{1}{d}}=1,
$$

which can be viewed as a lattice counterpart of Rényi information dimension [24].

\section{NONASYMPTOTIC ANALYSIS}

\section{A. Main result}

We now have all the tools required to provide a refined approximation to the minimum achievable coding rate at a given blocklength and a given low distortion.

Definition 4. An $(M, d, \epsilon)$ variable-length code for random variable $X \in \mathbb{R}^{n}$ is a pair of mappings $\mathrm{f}: \mathbb{R}^{n} \mapsto\{0,1\}^{\star}$ and $\mathrm{g}:\{0,1\}^{\star} \mapsto \mathbb{R}^{n}$ such that $\mathrm{d}(X, \mathrm{~g}(\mathrm{f}(X))) \leq d$ a.s. and $\mathbb{P}[\ell(\mathrm{f}(X))>\log M] \leq \epsilon$. The nonasymptotic fundamental limit is defined as

$$
R(n, d, \epsilon) \triangleq \frac{1}{n} \min \left\{\log M: \exists(M, d, \epsilon) \text { code for } X^{n} \in \mathbb{R}^{n}\right\}
$$

The main result of the section is stated next.

Theorem 8. Let $\mathrm{X} \in \mathbb{R}$ have $c_{1}|\mathrm{X}|+c_{0}$-regular density $f_{\mathrm{X}}$ such that $\mathbb{E}\left[\left\|\log f_{\mathrm{X}}(\mathrm{X})\right\|^{3}\right]<\infty$ and $\mathbb{E}\left[\mathrm{X}^{4}\right]<\infty$. For the compression of the source consisting of i.i.d. copies of $\mathrm{X}$ under mean-square error distortion, it holds that

$$
R(n, d, \epsilon)=\underline{R}(d)+\sqrt{\frac{\underline{\mathcal{V}}}{n}} Q^{-1}(\epsilon)+O(\sqrt{d})+O\left(\frac{\log n}{n}\right) .
$$

where $\underline{R}(d)$ and $\underline{\mathcal{V}}$ are given by (7) and (9), respectively. Moreover, (159) is attained by lattice quantization.

The proofs of the converse and the achievability parts of Theorem 8 are presented in Sections V-B and V-C below. Sources with memory are discussed in Section V-D. 


\section{B. Converse}

The observation that Shannon's lower bound can be obtained via (13) is key to the following result.

Theorem 9 (Converse). Assume that the distortion measure satisfies (17). For a nonnegative integer $k$, the encoded length of any variable-length code $(\mathrm{f}, \mathrm{g})$ operating at distortion $d$ must satisfy

$$
\begin{aligned}
& \mathbb{P}[\ell(\mathrm{f}(X)) \geq k] \\
\geq & \max _{\gamma>0}\left\{\mathbb{P}\left[\underline{\jmath}_{X}(X, d) \geq k+\gamma\right]-\exp (-\gamma)\right\},
\end{aligned}
$$

Proof: In [2, Appendix C], it is shown that for any $(g(\cdot), \lambda)$ satisfying constraint (14) it holds that $^{7}$

$$
\begin{aligned}
& \mathbb{P}[\ell(\mathrm{f}(X)) \geq k] \\
\geq & \max _{\gamma>0}\{\mathbb{P}[-\log g(X)-\lambda d \geq k+\gamma]-\exp (-\gamma)\},
\end{aligned}
$$

and (160) follows because $\underline{\jmath}_{X}(x, d)=-\log g(x)-\lambda d$ for the choice of $(\lambda, g)$ in Table I.

Assume now that $X$ in (160) is a vector of $n$ i.i.d. copies of $\mathrm{X}$, where random variable $\mathrm{X} \in \mathbb{R}$ has a density. ${ }^{8}$ Assume that the distortion measure is the MSE distortion in (20). Applying the BerryEsseén theorem to analyze the bound in (160) (see e.g. [2, Proof of Theorem 12] for the details on the technique), we conclude that

$$
n R(n, d, \epsilon) \leq n \underline{R}(d)+\sqrt{n \underline{\mathcal{V}}} Q^{-1}(\epsilon)+\frac{1}{2} \log n+O(1) .
$$

\section{Achievability}

For discrete random variable $S$, denote the information in $s$ by

$$
\imath_{S}(s) \triangleq \log \frac{1}{P_{S}(s)} .
$$

Consider the code $(f, g)$ which applies the optimal lossless codes to the output of lattice quantizer $\mathcal{C}$ (Fig. 2). Such a code assigns $k$ shortest binary strings to $k$ highest probability outcomes of $q_{\mathcal{C}}(X)$. Its excess-distortion probability is bounded by (e.g. [34])

$$
\mathbb{P}[\ell(f(X)) \geq k] \leq \mathbb{P}\left[\imath_{q_{\mathcal{C}}(X)}\left(q_{\mathcal{C}}(X)\right) \geq k\right]
$$

${ }^{7}$ In [2, Appendix C], the statement is made for the fixed-length code with excess distortion probability $\epsilon$, but it is not hard to see that it is equivalent to the variable-length setting with guaranteed distortion and excess length probability constraint of the present paper.

${ }^{8}$ Regularity is not required for the converse in (162). 
Assume further that $X$ is a vector of $n$ i.i.d. copies of $X$. Denote the constant

$$
a \triangleq \mathbb{E}\left[\mathrm{X}^{2}\right]+\sqrt{\operatorname{Var}\left[\mathrm{X}^{2}\right]}
$$

By Chebyshev's inequality,

$$
\mathbb{P}\left[\|X\|^{2}>n a\right] \leq \frac{1}{n}
$$

Using (84) and (90), we express the information in lattice quantizer output as

$$
\begin{aligned}
& \imath_{q_{\mathcal{C}}(X)}\left(q_{\mathcal{C}}(x)\right) \\
= & \log \frac{1}{f_{X}(x)}+\log \frac{1}{V_{\mathcal{C}}}+\log \frac{f_{X}(x)}{f_{X}\left(x+u_{x}\right)} \\
\leq & \log \frac{1}{f_{X}(x)}+\log \frac{1}{V_{\mathcal{C}}} \\
+ & r_{\mathcal{C}} \log e\left(c_{1}\|x\|+\sqrt{n} c_{0}+r_{\mathcal{C}}\right) \\
\leq & \log \frac{1}{f_{X}(x)}+\log \frac{1}{V_{\mathcal{C}}} \\
+ & r_{\mathcal{C}} \log e\left(\sqrt{n}\left(c_{0}+c_{1} \sqrt{a}\right)+r_{\mathcal{C}}\right)
\end{aligned}
$$

where $\left\|u_{x}\right\| \leq 2 r_{\mathcal{C}}$, and we used (98), (113) and the triangle inequality to upper bound the third term in (167). The last inequality holds with probability $1-\frac{1}{n}$ by (174).

Using (122), (114), (129), (134), and applying the Berry-Esseén bound to analyze (164) (see e.g. [2, Proof of Theorem 12] for the details on the technique), we conclude that

$$
\begin{aligned}
n R(n, d, \epsilon) & \leq n \underline{R}(d)+\sqrt{n \underline{\mathcal{V}}} Q^{-1}(\epsilon) \\
& +n \sqrt{d} \log e\left(c_{0}+\sqrt{a}+\sqrt{d}\right)+O(\log n)
\end{aligned}
$$

\section{Sources with memory}

The following result shows an estimate of the speed of convergence to $\underline{R}(d)$ for sources with memory.

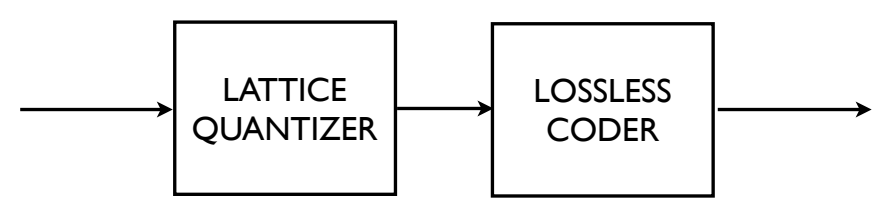

Fig. 2: Separated architecture of lattice quantization. 
Theorem 10. Let stationary ergodic process $X_{1}, X_{2}, \ldots$ be such that for each $n, X^{n}$ has $c_{1}\|x\|+\sqrt{n} c_{0^{-}}$ regular, log-concave density, and $\mathbb{E}\left[\|X\|^{2}\right] \leq n \mu$. For the compression of $X_{1}, X_{2}, \ldots$ under meansquare error distortion, it holds that

$$
R(n, d, \epsilon) \leq \underline{R}(d)+\frac{q(\epsilon)}{\sqrt{n}}+O(\sqrt{d})+O\left(\frac{\log n}{n}\right) .
$$

where $\underline{R}(d)$ is given in (7), and

$$
-\sqrt{\frac{1}{1-\epsilon}} \leq q(\epsilon) \leq \sqrt{\frac{1}{\epsilon}}
$$

Moreover, (171) is attained by lattice quantization.

Proof: For the achievability, note that trivially,

$$
\operatorname{Var}\left[\|X\|^{2}\right] \leq \mathbb{E}\left[\|X\|^{2}\right] \leq n \mu,
$$

so by Chebyshev's inequality,

$$
\mathbb{P}\left[\|X\|^{2}>2 n \mu\right] \leq \frac{1}{n}
$$

Therefore, as in (167)-(169),

$$
\begin{aligned}
& \imath_{q_{\mathcal{C}}(X)}\left(q_{\mathcal{C}}(X)\right) \leq_{\text {w.p. } 1-\frac{1}{n}} \log \frac{1}{f_{X}(X)}+\log \frac{1}{V_{\mathcal{C}}} \\
+ & r_{\mathcal{C}} \log e\left(\sqrt{n}\left(c_{0}+c_{1} \sqrt{2 \mu}\right)+r_{\mathcal{C}}\right) .
\end{aligned}
$$

On the other hand, by Chebyshev's inequality,

$$
\mathbb{P}\left[\log \frac{1}{f_{X}(X)}-h(X) \geq \sqrt{\frac{\operatorname{Var}\left[f_{X}(X)\right]}{\epsilon}}\right] \leq \epsilon .
$$

Letting in (164)

$$
k=h(X)+\sqrt{\frac{\operatorname{Var}\left[f_{X}(X)\right]}{\epsilon}}+\log \frac{1}{V_{\mathcal{C}}}+r_{\mathcal{C}} \log e\left(\sqrt{n}\left(c_{0}+c_{1} \sqrt{2 \mu}\right)+r_{\mathcal{C}}\right),
$$

we conclude using (176) and (122) that

$$
R(n, d, \epsilon) \leq \frac{k}{n}
$$

The achievability proof is complete upon applying the recent result of Fradelizi et al. [35, Theorem 2.3], which states that as long as $X \in \mathbb{R}^{n}$ has log-concave density,

$$
\operatorname{Var}\left[\log f_{X}(X)\right] \leq n
$$

For the converse, we weaken (160) by choosing $\gamma=\frac{1}{2} \log n$ :

$$
\mathbb{P}[\ell(f(X)) \geq k] \geq 1-\mathbb{P}\left[\underline{\jmath}_{X}(X, d)<k+\frac{1}{2} \log n\right]-\frac{1}{\sqrt{n}} .
$$


Let

$$
k=\underline{\mathbb{R}}_{X^{n}}(d)-\sqrt{\frac{\operatorname{Var}\left[f_{X}(X)\right]}{1-\epsilon-\frac{1}{\sqrt{n}}}}-\frac{1}{2} \log n,
$$

and note that the probability in the right side of (180) is upper-bounded by $1-\epsilon-\frac{1}{\sqrt{n}}$ by Chebyshev's inequality. We conclude that $\mathbb{P}[\ell(f(X)) \geq k] \geq \epsilon$, which implies the validity of the converse part of (171) when combined with (179).

Remark 16. Theorem 10 is easily generalized to stationary ergodic sources satisfying regularity conditions (130), (131) of Theorem 7, provided that the following additional condition holds for some $\sigma$,

$$
\mathbb{E}\left[\mathrm{v}^{2}\left(i \frac{\left\|x^{n}\right\|}{\sqrt{n}}\right)\right] \leq i \sigma^{2}, \quad i=1,2 ; n=1,2, \ldots,
$$

where $v$ is the function in (130).

\section{BEYOND MSE DISTORTION}

Sections III-V mainly focused on studying covering performance of lattices with respect the Euclidean norm. It turns out that those results generalize to a wider class of distortion measures.

In this section, we consider distortion measures of form

$$
\mathrm{d}\left(x^{n}, y^{n}\right)=\mathrm{d}\left(n^{-\frac{1}{p}}\left\|x^{n}-y^{n}\right\|_{p}\right)
$$

where $\|\cdot\|_{p}$ is the $L^{p}$ norm in $\mathbb{R}^{n}, 1 \leq p \leq \infty$, and $\mathrm{d}: \mathbb{R}^{+} \mapsto \mathbb{R}^{+}$is right-continuous. The scaling by $n^{-\frac{1}{p}}$ in (183) is chosen so that the distortion does not have a tendency to increase with increasing dimension $n$.

Example. Scaled $L^{p}$ norm distortion fits the framework of (183):

$$
\mathrm{d}\left(x^{n}, y^{n}\right)=n^{-\frac{s}{p}}\left\|x^{n}-y^{n}\right\|_{p}^{s},
$$

where $s>0$. Plugging $s=2$ and $p=2$ in (184) one recovers the MSE distortion measure. An interesting special case is that of the $L^{\infty}$ norm, which corresponds to the distortion measure

$$
\mathrm{d}\left(x^{n}, y^{n}\right)=\max _{1 \leq i \leq n}\left|x_{i}-y_{i}\right|^{s} .
$$

Section III generalizes completely, with an understanding that the balls and distances are now defined in terms of $L^{p}$ norm, rather than the Euclidean norm, while the regular density in Definition 1 is defined with respect to $L_{q}$ norm, where $\frac{1}{p}+\frac{1}{q}=1$. Proposition 1 generalizes verbatum via applying Hölder's inequality instead of Cauchy-Shwartz in (106). 
Proposition 2 generalizes as follows:

Proposition 3 (Generalization of Proposition 2). Consider lattice quantization of a continuous random vector $X^{n}$ with $h\left(X^{n}\right)>-\infty$ under distortion measure (183). Lattice $\epsilon$-entropy is characterized as

$$
\mathbb{L}_{X^{n}}(d)=h\left(X^{n}\right)+n \log \frac{1}{r(d)}-\frac{n}{p} \log n-\log b_{n, p}+\inf _{\mathcal{C}: r_{\mathcal{C}} \leq \sqrt[p]{n} r(d)}\left\{D\left(X^{n} \| X_{\mathcal{C}}^{n}\right)+n \log \rho_{\mathcal{C}}\right\}
$$

where

$$
r(d) \triangleq \inf \{r \geq 0: \mathrm{d}(r) \leq d\}
$$

and $b_{n, p}$ is the volume of a unit $L^{p}$ ball:

$$
b_{n, p} \triangleq \frac{\left(2 \Gamma\left(\frac{1}{p}+1\right)\right)^{n}}{\Gamma\left(\frac{n}{p}+1\right)} .
$$

If $\mathrm{d}: \mathbb{R}^{+} \mapsto \mathbb{R}^{+}$is invertible, then simply $r(d)=\mathrm{d}^{-1}(d)$. For example, the distortion in (184) corresponds to $\mathrm{d}(r)=r^{s}$ in the right side of (183); therefore, $r(d)=\sqrt[s]{d}$.

To study the covering efficiency of lattices with respect to d, in lieu of Rogers' Theorem 6 we invoke the following result of Rogers:

Theorem 11 (Rogers [9, Theorem 5.8], generalization of Theorem 6). For each $n \geq 3$, there exists an $n$-dimensional lattice $\mathcal{C}_{n}$ and constant $c$ with covering efficiency (with respect to any norm)

$$
n \log \rho_{\mathcal{C}_{n}} \leq \log n\left(\log _{2} n+c \log \log n\right)
$$

When particularized to the Euclidean norm, Theorem 11 presents a weakened version of Theorem 6. Next, we generalize Theorem 7 to the distortion measure in (183).

Theorem 12 (Generalization of Theorem 7). Let $p \geq 2$ and let $\frac{1}{p}+\frac{1}{q}=1$. Assume that for some integer $m \geq 0$ and scalar $\mu \geq 1$, the ergodic process $X_{1}, X_{2}, \ldots$ is such that:

(i) The density $f_{X^{n}}$ is $v_{n}$-regular (with respect to the $L_{q}$ norm) with

$$
v_{n}\left(x^{n}\right)=n^{\frac{1}{q}} \vee\left(n^{-\frac{1}{q}}\left\|x^{n}\right\|_{q}\right)
$$

where $\mathrm{v}: \mathbb{R}_{+} \mapsto \mathbb{R}_{+}$is convex and nondecreasing.

(ii) There exists $\mu<\infty$ such that

$$
\mathbb{E}\left[\mathrm{v}\left(i n^{-\frac{1}{q}}\left\|X^{n}\right\|_{q}\right)\right] \leq i \mu, \quad i=1,2 ; n=1,2, \ldots,
$$


Then, as $d \rightarrow 0$, the lattice rate-distortion function under distortion measure (183) expands as,

$$
L(d)=h+\log \frac{1}{r(d)}-\log c_{p}+O(r(d)),
$$

where $r(\cdot)$ is defined in (187), and for $p<\infty$

$$
c_{p} \triangleq 2 \Gamma\left(\frac{1}{p}+1\right)(p e)^{\frac{1}{p}},
$$

while $c_{\infty}=\lim _{p \rightarrow \infty} c_{p}=2$.

Proof: Applying Stirling's approximation to (188), we have

$$
\begin{aligned}
\log b_{n, p} & =n \log c_{p}-\frac{n}{p} \log n-\frac{1}{2} \log n+O(1), p<\infty \\
\log b_{n, \infty} & =n \log c_{\infty}=2 n .
\end{aligned}
$$

This establishes the asymptotics in $n$ of the first four terms in (186) and, therefore, the converse part of (192). To show the matching achievability bound, we need to bound the expression inside the infimum in the right side of (186) from above. The behavior of $\log \rho_{\mathcal{C}}$ in (186) is settled by Theorem 11. To bound $D\left(X^{n} \| X_{\mathcal{C}}^{n}\right)$, consider $v$-regular $f_{X}$ with $v(x)=v\left(\|x\|_{q}\right)$ that is convex and nondecreasing. We have for all $a$ and $b$ from the same lattice cell,

$$
\begin{aligned}
\left|\log f_{X}(a)-\log f_{X}(b)\right| & \leq \frac{\log e}{2}\left(v\left(\|a\|_{q}\right)+v\left(\|b\|_{q}\right)\right)\|a-b\|_{p} \\
& \leq \frac{\log e}{2}\left(v\left(\|a\|_{q}\right)+\frac{1}{2} v\left(2\|a\|_{q}\right)+\frac{1}{2} v\left(2\|a-b\|_{q}\right)\right)\|a-b\|_{p} \\
& \leq \frac{\log e}{2}\left(v\left(\|a\|_{q}\right)+\frac{1}{2} v\left(2\|a\|_{q}\right)+\frac{1}{2} v\left(2 n^{\frac{1}{q}-\frac{1}{p}}\|a-b\|_{p}\right)\right)\|a-b\|_{p} \\
& \leq \log e\left(v\left(\|a\|_{q}\right)+\frac{1}{2} v\left(2\|a\|_{q}\right)+\frac{1}{2} v\left(4 n^{\frac{1}{q}} r(d)\right)\right) n^{\frac{1}{p}} r(d)
\end{aligned}
$$

where (197) is by the Minkowski inequality and the convexity of $v,(198)$ is due to $\|\cdot\|_{q} \leq n^{\frac{1}{q}-\frac{1}{p}}\|\cdot\|_{p}$, valid for $0<q \leq p$, and (199) holds because $v$ is nondecreasing. Replacing $a$ and $b$ by $X$ and $X_{\mathcal{C}}$, taking an expectation of (199) and applying (190) and (191), we conclude

$$
\begin{aligned}
D\left(X^{n} \| X_{\mathcal{C}}^{n}\right) & \leq \log e\left(\mathbb{E}\left[\mathrm{v}\left(n^{-\frac{1}{q}}\|X\|_{q}\right)\right]+\frac{1}{2} \mathbb{E}\left[\mathrm{v}\left(2 n^{-\frac{1}{q}}\|X\|_{q}\right)\right]+\frac{1}{2} \mathrm{v}(4 r(d))\right) n r(d) \\
& \leq \log e\left(2 \mu+\frac{1}{2} \mathrm{v}(4 r(d))\right) n r(d)
\end{aligned}
$$

For the distortion measure in (184), we particularize (192) as

$$
L(d)=h+\frac{1}{s} \log \frac{1}{d}-\log c_{p}+O(\sqrt[s]{d}) .
$$


Plugging $s=2$ and $p=2$ into (202), one obtains $c_{p}=\sqrt{2 \pi e}$ and recovers (132). An interesting special case is that of $L^{\infty}$ norm, which corresponds to distortion measure $\mathrm{d}\left(x^{n}, y^{n}\right)=\max _{1 \leq i \leq n} \mid x_{i}-$ $\left.y_{i}\right|^{s}$ : since an $L^{\infty}$ ball is simply a cube, the cubic lattice quantizer attains the best covering efficiency. The crucial question is, of course, whether (192) attains Shannon's lower bound.

For the distortion in (184), a direct calculation using Table I shows that Shannon's lower bound is given by

$$
\begin{aligned}
\underline{\mathbb{R}}_{X^{n}}(d) & =h\left(X^{n}\right)+\frac{n}{s} \log \frac{1}{d}-\frac{n}{p} \log n-\log b_{n, p}+\frac{n}{s} \log \frac{n}{s e}-\log \Gamma\left(\frac{n}{s}+1\right) \\
& =h\left(X^{n}\right)+\frac{n}{s} \log \frac{1}{d}-n \log c_{p}+O(1), \quad n \rightarrow \infty
\end{aligned}
$$

Dividing by $n$ and taking a limit as $n \rightarrow \infty$, we conclude that

$$
\underline{R}(d)=h+\frac{1}{s} \log \frac{1}{d}-\log c_{p}
$$

Comparing to (202) we see that

$$
L(d)=\underline{R}(d)+O(\sqrt[s]{d})
$$

More generally, if $\mathrm{d}(\cdot)$ is differentiable at 0 and $0<\mathrm{d}^{\prime}(0)<\infty$, then by Taylor's approximation,

$$
r(d)=\frac{d}{\mathrm{~d}^{\prime}(0)}+o(d)
$$

If $\mathrm{d}^{\prime}(0)=\ldots=\mathrm{d}^{(s-1)}(0)=0$, and $0<\mathrm{d}^{(s)}(0)<\infty$, then

$$
r(d)=\sqrt[s]{\frac{s ! d}{\mathrm{~d}^{(s)}(0)}}+o(\sqrt[s]{d})
$$

Suppose further that $d(\cdot)$ in the right side of (183) satisfies Linkov's regularity conditions (28)-(30). Then, [5, Corollaries 1, 2] imply that

$$
\underline{\mathbb{R}}_{X^{n}}(d)=h\left(X^{n}\right)+\frac{n}{s} \log \frac{\mathrm{d}^{(s)}(0)}{s ! d}-n \log c_{p}+n o(1)+O(1),
$$

where $o(1)$ denotes a term that vanishes (uniformly in $n$ ) as $d \rightarrow 0$, and $O(1)$ denotes a term that is bounded by a constant. Comparing (209) to (192), (207), (208), we conclude that for a large class of non-MSE distortion measures, lattice quantization still approaches Shannon's lower bound:

$$
L(d)=\underline{R}(d)+o(1), d \rightarrow 0 .
$$

The main result of Section $\mathrm{V}$ generalizes as follows.

Theorem 13 (Generalization of Theorem 8). Let $p \geq 2$. Let $\mathrm{X} \in \mathbb{R}$ have $c_{1}|\mathrm{X}|+c_{0}$-regular density $f_{\mathrm{X}}$ such that $\mathbb{E}\left[\left\|\log f_{\mathrm{X}}(\mathrm{X})\right\|^{3}\right]<\infty$ and $\mathbb{E}\left[|\mathrm{X}|^{2 q}\right]<\infty$, where $\frac{1}{p}+\frac{1}{q}=1$. For the compression of the 
source consisting of i.i.d. copies of $\mathrm{X}$ under distortion measures of type (183), where $\mathrm{d}(\cdot)$ satisfies Linkov's conditions (28)-(30), it holds that

$$
R(n, d, \epsilon)=\underline{R}(d)+\sqrt{\frac{\underline{\mathcal{V}}}{n}} Q^{-1}(\epsilon)+o(1)+O\left(\frac{\log n}{n}\right),
$$

where and $\underline{R}(d)$ is defined in (146), $\underline{\mathcal{V}}$ is given by (9), and o(1) denotes a term that vanishes uniformly

in $n$ as $d \rightarrow 0$. For $\mathrm{d}$ in (183), o (1) can be refined to $O(\sqrt[s]{d})$. The result in (211) is attained by lattice quantization.

Proof: The converse results in Section V-B directly apply to all distortion measures of type (17) (and, therefore, to all distortion measures of type (183)). To show achievability of (211), we generalize (168) as,

$$
\imath_{q_{\mathcal{C}}(X)}\left(q_{\mathcal{C}}(x)\right) \leq \log \frac{1}{f_{X}(x)}+\log \frac{1}{V_{\mathcal{C}}}+n^{\frac{1}{p}} r(d) \log e\left(c_{1}\|x\|_{q}+n^{\frac{1}{q}} c_{0}+n^{\frac{1}{q}} r(d)\right),
$$

where we used (98) and (198). The rest of the proof follows that in Section V-C.

Note that (211) does not even require the distortion measure be separable.

\section{CONCLUSION}

Shannon's lower bound provides a powerful tool to study the rate-distortion function with respect to a difference distortion measure. We started the discussion by presenting the necessary and sufficient condition for Shannon's lower bound to be attained exactly (Theorem 3). As we saw in Theorem 4, all finite alphabet sources satisfy that condition for a range of low distortions. Whenever Shannon's lower bound is attained exactly, the d-tilted information in $x$ also admits a simple representation as the difference between the information in $x$ and a term that depends only on tolerated distortion $d$ (see (33)). This implies in particular that the rate-dispersion function of a discrete memoryless source with difference distortion measure is given simply by the varentropy of the source, as long as the target distortion is low enough.

Although continuous sources rarely attain Shannon's lower bound exactly, they often approach it at low distortions. For sources whose densities satisfy a smoothness condition, we showed a new bound on the output entropy of lattice quantizers in terms of the differential entropy of the source and the size of the lattice cell (Remark 4, Proposition 1). Leveraging that bound, we evaluated the best performance theoretically attainable by lattice quantization of ergodic sources in the limit of increasing dimension (Theorem 7). Finally, for high definition quantization of stationary memoryless sources with densities satisfying a smoothness condition, we showed an expansion of the minimum achievable source coding 
rate (Theorem 8). The appeal of the new expansion is its explicit nature and a simpler form compared to the more general result in [2]. A nonasymptotic Shannon's lower bound, valid for all $d$, is presented in Theorem 9. As (170) demonstrates, at low $d$, that bound is closely approached by lattice quantization. Going beyond memoryless sources, we showed that stationary ergodic sources satisfying a regularity assumption still attain that bound at speed $O\left(\frac{1}{\sqrt{n}}\right)$ with increasing blocklength (Theorem 10). The engineering implication is that a separated architecture of a lattice quantizer followed by a lossless coder displayed in Fig. 2 is nearly optimal.

\section{ACKNOWLEDGEMENT}

I would like to thank the Simons Institute for the Theory of Computing in Berkeley for providing the nurturing environment where this work started amid the discussions in the Spring of 2014; Dr. Tamás Linder for his insightful comments and in particular for describing an alternative way to obtain (149), now included in Remark 14; Dr. Tobias Koch for pointing out references [25], [32]; Dr. Yury Polyanskiy for mentioning the result in [35].

\section{APPENDIX A}

\section{AN UPPER BOUND ON THE ENTROPY OF AN INTEGER VECTOR}

Assume that vector $X$ has all integer components. The following bound is shown in [28, Lemma 3]:

$$
H(X) \leq L_{X}^{\star}+\log _{2}\left(L_{X}^{\star}+1\right)+\log _{2} e
$$

where $L_{X}^{\star}$ is the minimum average length of a lossless representation of $X$. Consider the following suboptimal lossless code: enumerate all integer $n$-vectors so that

$$
\mathbf{j} \preceq \mathbf{k} \text { if and only if }\|\mathbf{j}\| \leq\|\mathbf{k}\|
$$

and let the lossless representation of $X$ be the binary representation of the index number of $X$. Bounding $L_{X}^{\star}$ from above by the average length of this code, we have

$$
L_{X}^{\star} \leq \mathbb{E}\left[\log _{2} R_{n}(\|X\|)\right]
$$

where $R_{n}(r)$ is the number of integer-valued vectors with norm not exceeding $r$ :

$$
R_{n}(r) \triangleq \#\left\{\mathbf{k} \in \mathbb{Z}^{n}:\|\mathbf{k}\| \leq r\right\}
$$

To estimate $R_{n}(r)$, observe that $R_{n}(r)$ is upper bounded by the number of cubes with side 1 that intersect the ball of radius $r$. Since the diameter of cube of side 1 is $\sqrt{n}$, all those cubes are contained 
inside the augmented ball of radius $r+\sqrt{n}$. The number of volume-1 cubes that can be packed inside a ball is upper bounded by the volume of that ball; therefore,

$$
R_{n}(r) \leq b_{n}(r+\sqrt{n})^{n}
$$

A nonasymptotic Stirling's formula implies

$$
\log b_{n} \leq \frac{n}{2} \log \frac{2 \pi e}{n+2}-\frac{1}{2} \log (n+2)+\log \frac{e}{\sqrt{\pi}}
$$

so applying $\frac{z}{1+z} \leq \log _{e}(1+z)$ for $z>-1$ and (218) to (217), we obtain

$$
\begin{aligned}
\log R_{n}(r) & \leq \log b_{n}-\frac{n}{2} \log n+n \log \left(\frac{\sqrt{\sigma}}{\sqrt{n}}+1\right) \\
& \leq n \log \left(\frac{r}{\sqrt{n}}+1\right)+n \log \sqrt{2 \pi e}-\frac{1}{2} \log (n+2)-\log \sqrt{\pi}+\frac{2}{n+2} \log e .
\end{aligned}
$$

\section{REFERENCES}

[1] V. Kostina, "Data compression with low distortion and finite blocklength," in Proceedings 53rd Annual Allerton Conference on Communication, Control and Computing, Monticello, IL, Oct. 2015.

[2] V. Kostina and S. Verdú, "Fixed-length lossy compression in the finite blocklength regime," IEEE Transactions on Information Theory, vol. 58, no. 6, pp. 3309-3338, June 2012.

[3] R. Blahut, "Computation of channel capacity and rate-distortion functions," IEEE Transactions on Information Theory, vol. 18, no. 4, pp. $460-473,1972$.

[4] C. E. Shannon, "Coding theorems for a discrete source with a fidelity criterion," IRE Int. Conv. Rec., vol. 7, no. 1, pp. 142-163, Mar. 1959, reprinted with changes in Information and Decision Processes, R. E. Machol, Ed. New York: McGraw-Hill, 1960, pp. 93-126.

[5] Y. N. Linkov, "Evaluation of $\epsilon$-entropy of random variables for small $\epsilon$," Problems of Information Transmission, vol. 1, pp. 18-26, 1965.

[6] T. Linder and R. Zamir, "On the asymptotic tightness of the Shannon lower bound," IEEE Transactions on Information Theory, vol. 40, no. 6, pp. 2026-2031, 1994.

[7] J. H. Conway and N. Sloane, "Fast quantizing and decoding and algorithms for lattice quantizers and codes," IEEE Transactions on Information Theory, vol. 28, no. 2, pp. 227-232, 1982.

[8] A. Gersho and R. M. Gray, Vector quantization and signal compression. Springer Science \& Business Media, 2012 , vol. 159.

[9] C. A. Rogers, Packing and covering. Cambridge University Press, 1964, no. 54.

[10] A. Gersho, “Asymptotically optimal block quantization," IEEE Transactions on Information Theory, vol. 25, no. 4, pp. 373-380, 1979.

[11] R. Zamir and M. Feder, "On universal quantization by randomized uniform/lattice quantizers," IEEE Transactions on Information Theory, vol. 38, no. 2, pp. 428-436, 1992.

[12] T. T. Linder and K. K. Zeger, "Asymptotic entropy-constrained performance of tessellating and universal randomized lattice quantization," IEEE Transactions on Information Theory, vol. 40, no. 2, pp. 575-579, 1994.

[13] C. E. Shannon, "Probability of error for optimal codes in a Gaussian channel," Bell Syst. Tech. J., vol. 38, no. 3, pp. 611-656, 1959.

[14] W. R. Bennett, “Spectra of quantized signals,” Bell Systems Tech. J., vol. 27, pp. 446-472, 1948. 
[15] P. Zador, “Asymptotic quantization error of continuous signals and the quantization dimension," IEEE Transactions on Information Theory, vol. 28, no. 2, pp. 139-149, Mar 1982.

[16] I. Csiszár, "On an extremum problem of information theory," Studia Scientiarum Mathematicarum Hungarica, vol. 9, no. 1, pp. 57-71, Jan. 1974.

[17] T. Berger, Rate distortion theory. Prentice-Hall Englewood Cliffs, NJ, 1971.

[18] A. Gerrish and P. Schultheiss, "Information rates of non-Gaussian processes," IEEE Transactions on Information Theory, vol. 10, no. 4, pp. 265-271, Oct 1964.

[19] H. Whitney, Complex analytic varieties. Addison-Wesley Pub. Co., 1972.

[20] F. Zhang, Matrix theory: basic results and techniques. Springer Science \& Business Media, 2011.

[21] G. H. Golub and C. F. Van Loan, Matrix computations. JHU Press, 2012, vol. 3.

[22] V. Erokhin, "Epsilon-entropy of a discrete random variable," Theory of Probability and Applications, vol. 3, no. 1, pp. 97-100, 1958.

[23] I. Csiszár, "Generalized entropy and quantization problems," in Trans. Sixth Prague Conf Inform. Theory, Statist. Decision Functions, Random Processes. Prague, Czechoslovakia: Akademia, Sep. 1971, pp. 159-174.

[24] A. Rényi, "On the dimension and entropy of probability distributions," Acta Mathematica Academiae Scientiarum Hungarica, vol. 10, no. 1-2, pp. 193-215, 1959.

[25] T. Koch, “The shannon lower bound is asymptotically tight for sources with finite Rényi information dimension," arXiv preprint arXiv:1504.08245, 2015.

[26] Y. Polyanskiy and Y. Wu, "Wasserstein continuity of entropy and outer bounds for interference channels," arXiv preprint arXiv:1504.04419, 2015.

[27] E. C. Posner, E. R. Rodemich, and H. Rumsey, "Epsilon-entropy of stochastic processes," The Annals of Mathematical Statistics, vol. 38, no. 4, pp. 1000-1020, 1967.

[28] N. Alon and A. Orlitsky, "A lower bound on the expected length of one-to-one codes," IEEE Transactions on Information Theory, vol. 40, no. 5, pp. 1670-1672, Sep. 1994.

[29] A. Wyner, "An upper bound on the entropy series," Information and Control, vol. 20, no. 2, pp. 176-181, 1972.

[30] J. H. Conway and N. J. A. Sloane, Sphere packings, lattices and groups. Springer Science \& Business Media, 2013 , vol. 290.

[31] R. Zamir, Lattice Coding for Signals and Networks: A Structured Coding Approach to Quantization, Modulation, and Multiuser Information Theory. Cambridge University Press, 2014.

[32] T. Koch and G. Vazquez-Vilar, "Rate-distortion bounds for high-resolution vector quantization via Gibbs's inequality," arXiv preprint arXiv:1507.08349, 2015.

[33] R. Zamir and M. Feder, "On lattice quantization noise,” IEEE Transactions on Information Theory, vol. 42, no. 4, pp. 1152-1159, 1996.

[34] S. Verdú, "ELE528: Information theory lecture notes," Princeton University, 2009.

[35] M. Fradelizi, M. Madiman, and L. Wang, "Optimal concentration of information content for log-concave densities," arXiv preprint arXiv:1508.04093, 2015. 